\title{
Nutritional quality of commercial butters
}

\section{Qualidade nutricional de manteigas comerciais}

\author{
Maritiele Naissinger da Silva ${ }^{1 *}$ (D), Bruna Lago Tagliapietra ${ }^{2}$ (D), \\ Franciele Pozzebon Pivetta ${ }^{3}$, Vinicius do Amaral Flores ${ }^{4}$, \\ Neila Silvia Pereira dos Santos Richards ${ }^{4}$ \\ ${ }^{1}$ Centro Universitário UNIFASIPE, Curso de Nutrição, Sinop/MT - Brasil \\ ${ }^{2}$ Universidade Estadual de Campinas (UNICAMP), Departamento de Tecnologia de Alimentos, Campinas/SP . \\ Brasil \\ ${ }^{3}$ Instituto Federal de Educação, Ciência e Tecnologia de Santa Catarina (IFSC), Xanxerê/SC - Brasil \\ ${ }^{4}$ Universidade Federal de Santa Maria (UFSM), Departamento de Tecnologia e Ciência dos Alimentos, Santa \\ Maria/RS - Brasil
}

*Corresponding Author: Maritiele Naissinger da Silva, Universidade Federal de Santa Maria (UFSM),

Departamento de Tecnologia e Ciência dos Alimentos, Rua Antonio Botega, 270, CEP 97095-030, Santa Maria/RS,

Brasil, e-mail: maritielens@gmail.com

Cite as: M. N. Silva, Tagliapietra, B. L., Pivetta, F. P., Flores, V. A., \& Richards, N. S. P. S. (2021). Nutritional quality of commercial butters. Brazilian Journal of Food Technology, 24, e2020202. https://doi.org/10.1590/19816723.20220

\begin{abstract}
The consumption and consequent production of butter has increased considerably in recent years. In order to know the butters sold in Brazil, the study aimed to analyze butters of Brazilian, French, Italian and Argentine origin. The samples were analyzed for fat content, moisture, Defatted Dry Extract (DDE), and total acidity for comparison with Brazilian legislation. The levels of chlorides, protein, ash, total dry extract, water activity, color and determination of the lipid profile were also determined. In all analyses, there was a statistically significant difference between the samples. Some samples showed disagreement with the quality requirements recommended in Brazilian legislation for commercialization. Indeed, 10 samples did not present the minimum fat content required, varying from $68.53 \%$ to $77.31 \%$ in butters with salt and from $71.64 \%$ to $81.72 \%$ in those without salt. Eight samples presented humidity levels above the legal recommendations, varying from $17.05 \%$ to $20.28 \%$. All products were in agreement with acidity levels. The predominant fatty acids in all samples were myristic acid (C14: 0), palmitic acid (C16: 0), stearic acid (C18: 0) and oleic acid (C18: 1n9). The regional influence, breed and handling of the animals and season of the year in which the milk was obtained for butter production, were suggested to be the main reason for the physicalchemical difference found between the samples.
\end{abstract}

Keywords: Dairy products; Legislation of dairy products; Milk; Profile of fatty acids; Food quality.

\section{Resumo}

O consumo e, consequentemente, a produção de manteiga têm aumentado consideravelmente nos últimos anos. Para conhecer as manteigas comercializadas no Brasil, o estudo teve como objetivo analisar manteigas de origem brasileira, francesa, italiana e argentina. As amostras foram analisadas quanto ao teor de gordura, à umidade, ao extrato seco desengordurado e à acidez total, para comparação com a legislação brasileira. Também foram determinados os teores de cloretos, proteínas, cinzas, extrato seco total, atividade de água, cor e perfil lipídico. Em todas as análises, houve diferença significativa entre as amostras. Algumas amostras apresentaram desacordo com 
os requisitos de qualidade recomendados pela legislação brasileira para comercialização. Dez amostras não apresentaram o teor mínimo de gordura exigido, variando de $68,53 \%$ a 77,31\% nas manteigas com sal e de $71,64 \%$ a $81,72 \%$ nas manteigas sem sal. Oito amostras estavam acima do permitido para umidade, variando de $17,05 \%$ a 20,28\%, e todas estavam de acordo em relação à acidez. Os ácidos graxos predominantes em todas as amostras foram ácido mirístico (C14:0), ácido palmítico (C16:0), ácido esteárico (C18:0) e ácido oleico (C18:1n9). A influência regional, a raça do animal, o manejo dos animais e a época do ano em que o leite foi obtido para a produção das manteigas são considerados os principais motivos das diferenças físico-químicas encontradas entre as amostras.

Palavras-chave: Produtos lácteos; Legislação de lácteos; Leite; Perfil dos ácidos graxos; Qualidade alimentar.

\section{Introduction}

Dairy products are among the most consumed foods worldwide and are studied for their own benefits to human health. Some components of milk fat may have hypocholesterolemic, anticancer and antidiabetogenic effects (Verruck et al., 2019). Butter is an important derivative in the dairy industry owing to its sensory attributes, as well as its versatility that meets the needs of a wide range of consumers (Soundous et al., 2019) at the consumer's table.

Butter is a mechanical agglomeration of fat that contains at least $80 \%$ of fat, at most $16 \%$ of water, $0.5 \%$ of protein as well as $0.5 \%$ of carbohydrates, in addition to fat-soluble vitamins and minerals, making it a product of high caloric and nutritive content (Brasil, 1996; Méndez-Cid et al., 2017). Butter consumption has increased in the last few years, since the characteristics and quality of the sample has been the subject of many researches (Parmar et al., 2021; Nilchian et al., 2020), mainly seeing that its benefits are being studied and exposed.

According to Regulamento da Inspeção Industrial e Sanitária de Produtos de Origem Animal (RIISPOA) (Brasil, 2017), translated into English "the Regulation of Industrial and Sanitary Inspection of Products of Animal Origin", and approved by Decree No. 9.013, of March 29, 2017 and Ordinance No. 146, of March 7, 1996, which could establishe the Technical Regulation on Identity and Quality of Butter (in Portuguese Regulamento Técnico de Identidade e Qualidade (RTIQ)), defines butter as the fat milk product, which is found in the beat and the malaxation, with or without biological alteration of the cream milk, concerning a specific process.

The fat is an important component for diets (Méndez-Cid et al., 2017), not only for its vital function, but also as a source of cellular energy and for its energy content, as well as for being a vehicle of fat-soluble vitamins and providing fatty acids (Chamberlain et al., 2016). For a long time, foods of animal origin, such as butter, have been found in human life as precursors of cardiovascular diseases due to saturated fatty acid and cholesterol. However, studies have contributed to the reevaluation of dairy products, owing to the potential benefits of milk fat, such as the presence of Conjugated Linoleic Acid (CLA) (Yang et al., 2018; Zoidis et al., 2018; Méndez-Cid et al., 2017; Erkaya et al., 2015; Ledoux et al., 2005), that are important for human health, as they provide reduction in body fat accumulation, and also have anticarcinogenic action, antiatherogenic, antidiabetogenic and immunomodulating properties (Lopes et al., 2011; Santos-Zago et al., 2008; Dewhurst et al., 2006).

In the interest of seeking information on the quality of butter Brazilian and imported, which is little studied, and draw a characterization of this product, this study aimed to determine the physical and chemical parameters and fatty acids profile from Brazilian, French, Italian and Argentine butters marketed in Brazil and compare with the requirements of the Brazilian technical regulation. 


\section{Material and methods}

Butters were purchased in hypermarkets and transported to the place where they were analyzed inside thermal boxes containing synthetic ice to guarantee the stability of the products, being kept at a temperature of up to $5{ }^{\circ} \mathrm{C}$ or as established in the labeling of the products. During the analysis period, the butters were stored in refrigeration, maintaining the maximum temperature of $5^{\circ} \mathrm{C}$.

It was analyzed 29 samples of Brazilian-made and imported butters, salted and unsalted, from different commercial brands, of which 18 were Brazilian, with six products from Rio Grande do Sul, five from São Paulo, four from Paraná and three from Minas Gerais states, eight from France, two from Argentina and one from Italy. All samples were submitted to be analyzed with a maximum of 30 days after the date of manufacture. The validity periods of the 29 samples ranged from four to eight months. After opening the products, the analysis was completed in a maximum period of seven days.

The butters were divided into salted (14) and unsalted (15). To identify the samples, letters related to the countries of origin were used (B, F, A, I for Brazil, France, Argentina and Italy, respectively). In the case of Brazilian samples, the letters regarding states were used (PR, MG, RS, SP for Paraná, Minas Gerais, Rio Grande do Sul and São Paulo, respectively), even as it was added the letter S for the salty samples.

The samples were submitted to physical-chemical analysis to determine the levels of fat, moisture, Defatted Dry Extract (DDE) and total acidity, according to the requirements of Technical Regulation no. 146 of 1996 (Brasil, 1996), and to complement the characterization, the determination of the protein, ash, chlorides and water activity contents was determined. All analyses were performed in triplicates.

For the determination of fat content, the method used was based on the extraction of cold fat using a mixture of three solvents, chloroform-methanol-water, in proportion in which the three solvents form a homogeneous solution (Bligh \& Dyer, 1959). The moisture content was determined by the oven drying method at $105^{\circ} \mathrm{C}$ until constant weight was obtained (Association of Official Analytical, 2005). The total acidity was determined by volumetric titration with $0.1 \mathrm{~N}$ sodium hydroxide solution $(\mathrm{NaOH})$ (Association of Official Analytical, 2005). The determination of the protein content was performed by the method of micro Kjeldahl (Association of Official Analytical, 2005). The method used to determine the ash content was incineration, in muffle at $550^{\circ} \mathrm{C}$ until total burning of the organic matter (Association of Official Analytical, 2005). Chloride content was determined by solvent digestion and subsequent titration with $0.1 \mathrm{~mol}^{\mathrm{L}} \mathrm{L}^{-1}$ silver nitrate (Association of Official Analytical, 2005). The water activity was measured directly through a water activity analyzer of the AquaLab brand, model $4 \mathrm{TEV}$, at constant temperature of $25^{\circ} \mathrm{C}$.

Color analysis on the surface of refrigerated butters $\left(5^{\circ} \mathrm{C}\right)$ was carried out, in six times, according to the system of the International Commission of L'Éclairage (CIE), using the $L^{*}, a^{*}$ and $b^{*}$ coordinates through the Minolta ${ }^{\circledR}$ colorimeter (model CM-700d, Konica Minolta, Osaka, Japan), with illuminant A, $10^{\circ}$ angle, reflectance measurement included (SCI), with a measuring area of $8 \mathrm{~mm}$. The results were expressed as $L^{*}$ (luminosity), $a^{*}$ (where $-\mathrm{a}^{*}$ represented direction to green and $+\mathrm{a}^{*}$ direction to red), $b^{*}$ (where $-\mathrm{b}^{*}$ represented blue direction and $+b^{*}$ yellow direction).

The determination of the fatty acid profile of the butter samples was carried out in triplicate and followed the recommendations proposed by Hara \& Radin (1978) for the extraction of lipids, the samples being dried and stored under freezing $\left(-20^{\circ} \mathrm{C}\right)$ until proceeding to the methylation step and determination of the fatty acid profile. Lipid methylation followed the methodology proposed by Christie (1982) and then subjected to chromatographic analysis.

The quantification of fatty acid methyl esters was determined according to Visentainer (2012), by injecting $1 \mu \mathrm{L}$ into a Zebron $100 \%$ polyethylene glycol capillary column $(60 \mathrm{~m} \times 0.32 \mathrm{~mm}$ i.d. $\times 0.25 \mu \mathrm{m}$ film thickness; ZB-WAX plus, Phenomenex, Torrance, CA). The equipment used was an Agilent Technologies gas chromatograph, series 6890N, equipped with Flame Ionization Detector (FID), Split injector and capillary silica fused silica, with the dimensions of $60 \mathrm{~m}$ in length, $0.25 \mathrm{~mm}$ internal diameter and $0.2 \mu \mathrm{m}$ film 
thickness. Nitrogen was used as the entrainment gas. The sample volume injected was $1 \mu \mathrm{L}$. Fatty acids were identified by comparison to retention times of reference standards. The internal standard added was methyl tricosanoate in isooctane.

The stratification of the results was performed in Microsoft Excel ${ }^{\circledR}$, and analyzed according to a completely randomized design, applying descriptive analysis, Analysis of Variance (ANOVA) and the Tukey's test for multiple comparisons between the means, at a level of 5\% significance. The statistical program used was SPSS ${ }^{\circledR}$ version 15.0. The development of the study took place at the Federal University of Santa Maria (Universidade Federal de Santa Maria (UFSM)), in the Department of Food Science and Technology.

\section{Results and discussion}

In Brazil, for production and marketing, butter must comply with the RTIQ, which establishes the quality parameters for butter, recommending a minimum fat content of $82 \%$, for unsalted butter, and $80 \%$ for butter with salt. Maximum moisture should be $16 \%$, DDE with a maximum limit of $2 \%$ and acidity should not exceed $3 \mathrm{mmol} / 100 \mathrm{~g}$ of fat (Brasil, 1996).

In the statistical treatment of the data, the samples were distinguished into: samples with added salt; and samples not added salt. None of the samples had food dye added to their composition, according to labeling.

Table 1 presents the results of the physical-chemical analysis of the salt butters, totaling 14 samples, of which five samples (three from French, one from Argentina and one Brazilian) had a lower fat content than that recommended by Brazilian legislation and higher moisture content. Only one sample (French) presented adequate values for DDE.

The added salt content should not exceed 2\%, according to Ordinance 146/1996 (Brasil, 1996), and all the samples denominated "salted", according to the labeling information, did not present higher levels for the analysis of chlorides, ranging from $0.54 \%$ to $1.80 \%$ (Table 1), all of which were in accordance with Brazilian legislation. Regarding protein contents, the samples with salt obtained minimum values of $0.34 \%$ and maximum of $2.13 \%$. Salt-free samples had levels ranging from $0.36 \%$ to $2.01 \%$ of protein. The ash contents ranged from 0.02 to 2.28 for the samples with salt and from 0.02 to 0.55 for the unsalted butters.

Table 1. Average results of the physical-chemical analysis of the samples of national-made butter and imported trademarks, salted.

\begin{tabular}{|c|c|c|c|c|c|c|c|c|c|c|c|c|}
\hline \multirow{2}{*}{ Sample } & \multirow{2}{*}{$\begin{array}{l}\text { Fats } \\
(\%)\end{array}$} & \multirow{2}{*}{$\begin{array}{c}\text { Moisture } \\
\text { (\%) }\end{array}$} & \multirow{2}{*}{$\begin{array}{c}\text { Chlorides } \\
(\%)\end{array}$} & \multirow{2}{*}{$\begin{array}{c}\text { Acidity } \\
(\mathrm{mmol} / \mathbf{1 0 0} \mathrm{g})\end{array}$} & \multirow{2}{*}{$\begin{array}{c}\text { DDE } \\
(\%)\end{array}$} & \multirow{2}{*}{$\begin{array}{c}\text { Protein } \\
(\%)\end{array}$} & \multirow{2}{*}{$\begin{array}{c}\text { Ashes } \\
(\%)\end{array}$} & \multirow{2}{*}{$\begin{array}{l}\text { Aw } \\
(\%)\end{array}$} & \multirow{2}{*}{$\begin{array}{l}\text { TDE } \\
(\%)\end{array}$} & \multicolumn{3}{|c|}{ Color } \\
\hline & & & & & & & & & & $a^{*}$ & $\mathbf{b}^{*}$ & $\mathbf{L}^{*}$ \\
\hline B-RS-S & $82.93 \pm 0.3^{\mathrm{ab}}$ & $14.34 \pm 0.3^{\text {def }}$ & $0.76 \pm 0.0^{\mathrm{g}}$ & $1.90 \pm 0.7^{\mathrm{abc}}$ & $2.73 \pm 0.5^{\mathrm{fg}}$ & $0.34 \pm 0.0^{\mathrm{g}}$ & $0.99 \pm 0.0^{\mathrm{d}}$ & $0.95 \pm 0.0^{\mathrm{a}}$ & $85.66 \pm 0.3^{\mathrm{bcd}}$ & $-5.13^{\mathrm{h}}$ & $30.45^{\mathrm{f}}$ & $88.83^{\mathrm{abc}}$ \\
\hline B-PR-S & $80.69 \pm 0.2^{\mathrm{de}}$ & $14.14 \pm 0.2^{\text {def }}$ & $1.56 \pm 0.0^{\mathrm{bc}}$ & $1.60 \pm 0.1^{\mathrm{c}}$ & $5.17 \pm 0.4^{\text {cde }}$ & $1.49 \pm 0.2^{\mathrm{bc}}$ & $1.53 \pm 0.0^{\mathrm{c}}$ & $0.96 \pm 0.0^{\mathrm{a}}$ & $85.86 \pm 0.2^{\mathrm{bcd}}$ & $-8.09^{\mathrm{ab}}$ & $40.48^{\mathrm{b}}$ & $83.14^{\text {de }}$ \\
\hline B-PR-S & $81.18 \pm 0.7^{\text {cde }}$ & $13.32 \pm 0.4^{\mathrm{fg}}$ & $1.59 \pm 0.0^{\mathrm{bc}}$ & $1.71 \pm 0.1^{\mathrm{bc}}$ & $5.50 \pm 0.9^{\text {bcde }}$ & $1.11 \pm 0.0^{\text {cde }}$ & $1.50 \pm 0.1^{\mathrm{c}}$ & $0.96 \pm 0.0^{\mathrm{a}}$ & $86.68 \pm 0.4^{\mathrm{ab}}$ & $-6.68^{\mathrm{ef}}$ & $33.56^{\mathrm{e}}$ & $91.39^{\mathrm{ab}}$ \\
\hline B-RS-S & $80.58 \pm 0.1^{\mathrm{e}}$ & $13.56 \pm 0.2^{\operatorname{defg}}$ & $0.54 \pm 0.1^{\mathrm{h}}$ & $1.74 \pm 0.2^{\mathrm{bc}}$ & $5.87 \pm 0.4^{\mathrm{bcd}}$ & $1.73 \pm 0.2^{\mathrm{ab}}$ & $0.48 \pm 0.0^{\mathrm{e}}$ & $0.96 \pm 0.0^{\mathrm{a}}$ & $86.44 \pm 0.2^{\mathrm{abcd}}$ & $-7.41^{\mathrm{dd}}$ & $37.62^{\mathrm{c}}$ & $89.59^{\mathrm{abc}}$ \\
\hline B-MG-S & $82.44 \pm 0.5^{\mathrm{abc}}$ & $14.86 \pm 0.7^{\mathrm{de}}$ & $1.54 \pm 0.0^{\mathrm{bcd}}$ & $1.91 \pm 0.1^{\mathrm{abc}}$ & $2.70 \pm 0.3^{\text {fg }}$ & $0.89 \pm 0.1^{\mathrm{ef}}$ & $1.46 \pm 0.2^{\mathrm{c}}$ & $0.95 \pm 0.0^{\mathrm{a}}$ & $85.14 \pm 0.7^{\mathrm{cd}}$ & $-8.59^{\mathrm{a}}$ & $43.06^{\mathrm{a}}$ & $83.51^{\text {de }}$ \\
\hline B-SP-S & $83.51 \pm 0.4^{\mathrm{a}}$ & $12.19 \pm 0.7^{\mathrm{g}}$ & $1.37 \pm 0.0^{\mathrm{e}}$ & $1.53 \pm 0.4^{\mathrm{c}}$ & $4.30 \pm 0.9^{\mathrm{def}}$ & $1.69 \pm 0.2^{\mathrm{ab}}$ & $1.31 \pm 0.0^{\mathrm{c}}$ & $0.96 \pm 0.0^{\mathrm{a}}$ & $87.81 \pm 0.7^{\mathrm{a}}$ & $-7.08^{\text {cde }}$ & $36.97^{\mathrm{c}}$ & $82.82^{\mathrm{de}}$ \\
\hline F-S & $82.84 \pm 0.2^{\mathrm{ab}}$ & $13.50 \pm 0.4^{\text {efg }}$ & $1.80 \pm 0.0^{\mathrm{a}}$ & $1.87 \pm 0.0^{\mathrm{bc}}$ & $3.66 \pm 0.3^{\mathrm{efg}}$ & $1.74 \pm 0.3^{\mathrm{ab}}$ & $1.40 \pm 0.3^{\mathrm{c}}$ & $0.96 \pm 0.0^{\mathrm{a}}$ & $86.50 \pm 0.4^{\mathrm{abc}}$ & $-6.48^{\mathrm{f}}$ & $35.68^{\text {cde }}$ & $87.77^{\mathrm{abc}}$ \\
\hline B-SP-S & $83.23 \pm 0.1^{\mathrm{ab}}$ & $14.88 \pm 0.1^{\mathrm{de}}$ & $1.10 \pm 0.1^{\mathrm{f}}$ & $2.05 \pm 0.1^{\mathrm{ab}}$ & $1.89 \pm 0.0^{\mathrm{g}}$ & $0.56 \pm 0.0^{\mathrm{fg}}$ & $1.25 \pm 0.0^{\mathrm{cd}}$ & $0.95 \pm 0.0^{\mathrm{a}}$ & $85.12 \pm 0.1^{\mathrm{cd}}$ & $-6.75^{\text {ef }}$ & $36.04^{\text {cd }}$ & $80.46^{\mathrm{fg}}$ \\
\hline B-RS-S & $82.06 \pm 0,7^{\text {bod }}$ & $14.80 \pm 0.5^{\text {def }}$ & $1.67 \pm 0.0^{\mathrm{ab}}$ & $1.72 \pm 0.1^{\mathrm{bc}}$ & $3.14 \pm 0.2^{\mathrm{fg}}$ & $1.04 \pm 0.0^{\text {cde }}$ & $1.96 \pm 0.0^{\mathrm{b}}$ & $0.96 \pm 0.0^{\mathrm{a}}$ & $85.20 \pm 0.5^{\mathrm{bcd}}$ & $-7.49^{\mathrm{bc}}$ & $36.50^{\mathrm{c}}$ & $78.53^{\mathrm{g}}$ \\
\hline
\end{tabular}


Table 1. Continued..

\begin{tabular}{|c|c|c|c|c|c|c|c|c|c|c|c|c|}
\hline F-S & $73.35 \pm 0.5^{\mathrm{g}}$ & $19.18 \pm 0.5^{\mathrm{ab}}$ & $1.64 \pm 0.1^{\mathrm{abc}}$ & $2.06 \pm 0.1^{\mathrm{ab}}$ & $7.47 \pm 0.5^{\mathrm{b}}$ & $1.16 \pm 0.2^{\text {cde }}$ & $0.71 \pm 0.0^{\mathrm{e}}$ & $0.96 \pm 0.0^{\mathrm{a}}$ & $80.82 \pm 0.5^{\mathrm{fg}}$ & $-3.45^{f}$ & $21.72^{\mathrm{g}}$ & $92.59^{\mathrm{a}}$ \\
\hline F-S & $77.31 \pm 0.4^{\mathrm{f}}$ & $18.67 \pm 0.2^{\mathrm{b}}$ & $1.50 \pm 0.1^{\text {bode }}$ & $1.79 \pm 0.1^{\mathrm{bc}}$ & $4.02 \pm 0.3^{\mathrm{def}}$ & $1.65 \pm 0.2^{\mathrm{b}}$ & $2.28 \pm 0.1^{\mathrm{a}}$ & $0.95 \pm 0.0^{\mathrm{a}}$ & $81.33 \pm 0.2^{\mathrm{f}}$ & $-6.97^{\mathrm{cd}}$ & $33.81^{\mathrm{de}}$ & $88.45^{\mathrm{abc}}$ \\
\hline B-RS-S & $72.56 \pm 0.4^{\mathrm{g}}$ & $20.28 \pm 0.4^{\mathrm{a}}$ & $1.61 \pm 0.1^{\mathrm{bc}}$ & $2.07 \pm 0.1^{\mathrm{ab}}$ & $7.15 \pm 0.7^{\mathrm{bc}}$ & $2.13 \pm 0.2^{\mathrm{a}}$ & $1.01 \pm 0.0^{\mathrm{d}}$ & $0.97 \pm 0.0^{\mathrm{a}}$ & $79.72 \pm 0.4^{\mathrm{g}}$ & $-7.18^{\text {cde }}$ & $36.81^{\mathrm{c}}$ & $83.95^{\text {cde }}$ \\
\hline A-S & $71.09 \pm 0.9^{\mathrm{h}}$ & $15.00 \pm 0.6^{\mathrm{d}}$ & $1.38 \pm 0.0^{\mathrm{de}}$ & $2.29 \pm 0.0^{\mathrm{a}}$ & $13.91 \pm 0.9^{\mathrm{a}}$ & $0.93 \pm 0.0^{\mathrm{def}}$ & $1.84 \pm 0.0^{\mathrm{b}}$ & $0.97 \pm 0.0^{\mathrm{a}}$ & $85.00 \pm 0.6^{\mathrm{d}}$ & $-6.82^{\mathrm{df}}$ & $35.73^{\text {cde }}$ & $86.30^{\text {bcd }}$ \\
\hline F-S & $68.53 \pm 0.3^{\mathrm{i}}$ & $17.05 \pm 0.9^{\mathrm{c}}$ & $1.49 \pm 0.0^{\text {cde }}$ & $1.55 \pm 0.0^{c}$ & $14.43 \pm 0.9^{\mathrm{a}}$ & $1.36 \pm 0.1^{\mathrm{bod}}$ & $0.02 \pm 0.0^{\mathrm{f}}$ & $0.96 \pm 0.0^{\mathrm{a}}$ & $82.95 \pm 0.1^{\mathrm{e}}$ & $-6.39^{f}$ & $30.43^{\mathrm{f}}$ & $82.64^{\text {ffg }}$ \\
\hline C.V. $(\%)$ & 0.59 & 3.23 & 4.05 & 7.48 & 11.41 & 11.90 & 7.41 & 1.27 & 0.59 & 6.61 & 4.62 & 5.05 \\
\hline
\end{tabular}

Results presented by the average of the triplicates followed \pm Standard Deviation. Color presents the average values for a six-fold. The values in the same column, with equal letters, were not significant at the 5\% level. Tags: DDE (Defatted Dry Extract); Aw (Water activity); TDE (Total Dry Extract); C.V. (Coefficient of Variation).

Table 2 shows the results of the physical-chemical analysis of the 15 samples of unsalted butter, with five samples (three from French, one from Argentina and one Brazilian) being in disagreement with RTIQ for fat content and three samples (two Brazilian and French) presented higher values for humidity. For DDE, eight samples had adequate values of at most $2 \%$.

Table 2. Average results of the physical-chemical analysis of the butter samples of national and imported trademarks, unsalted.

\begin{tabular}{|c|c|c|c|c|c|c|c|c|c|c|c|c|}
\hline \multirow{2}{*}{ Samples } & \multirow{2}{*}{$\begin{array}{l}\text { Fats } \\
(\%)\end{array}$} & \multirow{2}{*}{$\begin{array}{c}\text { Moisture } \\
(\%)\end{array}$} & \multirow{2}{*}{$\begin{array}{c}\text { Chlorides } \\
(\%)\end{array}$} & \multirow{2}{*}{$\begin{array}{c}\text { Acidity } \\
\text { (mmol/100 g) }\end{array}$} & \multirow{2}{*}{$\begin{array}{c}\text { DDE } \\
(\%)\end{array}$} & \multirow{2}{*}{$\begin{array}{c}\text { Protein } \\
(\%)\end{array}$} & \multirow{2}{*}{$\begin{array}{c}\text { Ashes } \\
(\%)\end{array}$} & \multirow{2}{*}{$\begin{array}{l}\text { Aw } \\
(\%)\end{array}$} & \multirow{2}{*}{$\begin{array}{l}\text { TDE } \\
(\%)\end{array}$} & \multicolumn{3}{|c|}{ Color } \\
\hline & & & & & & & & & & $a^{*}$ & $\mathbf{b}^{*}$ & $\mathbf{L}^{*}$ \\
\hline B-RS & $84.87 \pm 0.1^{\mathrm{ab}}$ & $13.63 \pm 0.5^{\mathrm{ef}}$ & $0.05 \pm 0.0^{\mathrm{cd}}$ & $1.72 \pm 1.4^{\mathrm{ef}}$ & $1.49 \pm 0.4^{\mathrm{ef}}$ & $0.55 \pm 0.0^{\mathrm{d}}$ & $0.49 \pm 0.0^{\mathrm{ab}}$ & $0.98 \pm 0.0^{\mathrm{abc}}$ & $86.37 \pm 0.4^{\mathrm{bc}}$ & $-7.60^{\mathrm{bc}}$ & $37.62^{\mathrm{bc}}$ & $89.44^{\mathrm{ab}}$ \\
\hline B-SP & $83.70 \pm 0.6^{\mathrm{bcd}}$ & $14.27 \pm 0.4^{\mathrm{def}}$ & $0.03 \pm 0.0^{\mathrm{cd}}$ & $2.55 \pm 0.4^{\mathrm{b}}$ & $2.03 \pm 0.3^{\text {def }}$ & $0.63 \pm 0.0^{\mathrm{d}}$ & $0.50 \pm 0.0^{\mathrm{ab}}$ & $0.97 \pm 0.0^{\mathrm{a}}$ & $85.73 \pm 0.3^{\mathrm{bcd}}$ & $-6.58^{\mathrm{ef}}$ & $31.76^{\text {fg }}$ & $92.57^{\mathrm{a}}$ \\
\hline B-PR & $79.38 \pm 0.2^{\mathrm{g}}$ & $13.87 \pm 0.2^{\mathrm{ef}}$ & $0.05 \pm 0.0^{\mathrm{cd}}$ & $1.67 \pm 0.2^{\text {efg }}$ & $6.75 \pm 0.3^{\mathrm{b}}$ & $0.54 \pm 0.0^{\mathrm{d}}$ & $0.45 \pm 0.0^{\mathrm{b}}$ & $0.96 \pm 0.0^{\mathrm{ab}}$ & $86.13 \pm 0.2^{\mathrm{bc}}$ & $-7.10^{\mathrm{d}}$ & $34.92^{\mathrm{de}}$ & $91.06^{\mathrm{ab}}$ \\
\hline I & $82.98 \pm 0.2^{\mathrm{de}}$ & $14.66 \pm 0.4^{\mathrm{de}}$ & $0.02 \pm 0.0^{\mathrm{d}}$ & $2.27 \pm 1.2^{\mathrm{bc}}$ & $2.36 \pm 0.4^{\text {cdef }}$ & $0.70 \pm 0.0^{\text {cd }}$ & $0.55 \pm 0.0^{\mathrm{a}}$ & $0.96 \pm 0.0^{\mathrm{a}}$ & $85.34 \pm 0.3^{\text {cd }}$ & $-3.48^{\mathrm{k}}$ & $20.88^{j}$ & $\begin{array}{c}87.20^{\mathrm{ab}} \\
\mathrm{c}\end{array}$ \\
\hline B-SP & $82.42 \pm 0.3^{\text {ef }}$ & $14.20 \pm 0.8^{\mathrm{ef}}$ & $0.06 \pm 0.0^{\mathrm{bc}}$ & $1.50 \pm 0.9^{\mathrm{fgh}}$ & $3.38 \pm 0.9^{\text {cd }}$ & $0.36 \pm 0.0^{\mathrm{d}}$ & $0.55 \pm 0.0^{\mathrm{a}}$ & $0.97 \pm 0.0^{\mathrm{bcd}}$ & $85.80 \pm 0.8^{\mathrm{bc}}$ & $-4.69^{i}$ & $26.25^{\mathrm{i}}$ & $91.95^{\mathrm{a}}$ \\
\hline B-RS & $82.75 \pm 0.6^{\text {def }}$ & $16.14 \pm 0.0^{\mathrm{bc}}$ & $0.04 \pm 0.0^{\mathrm{cd}}$ & $1.71 \pm 0.6^{\mathrm{ef}}$ & $1.10 \pm 0.5^{\mathrm{f}}$ & $0.55 \pm 0.0^{\mathrm{d}}$ & $0.07 \pm 0.0^{\mathrm{e}}$ & $0.96 \pm 0.0^{\mathrm{ab}}$ & $83.86 \pm 0.0^{\text {ef }}$ & $-7.25^{\mathrm{cd}}$ & $36.32^{\mathrm{cd}}$ & $85.83^{\mathrm{bc}}$ \\
\hline B-MG & $82.54 \pm 0.5^{\text {def }}$ & $16.63 \pm 0.3^{b}$ & $0.05 \pm 0.0^{\mathrm{cd}}$ & $1.25 \pm 1.14^{\mathrm{h}}$ & $0.83 \pm 0.3^{\mathrm{f}}$ & $0.60 \pm 0.1^{\mathrm{d}}$ & $0.03 \pm 0.0^{\mathrm{e}}$ & $0.96 \pm 0.0^{\mathrm{cd}}$ & $83.37 \pm 0.2^{\mathrm{f}}$ & $-6.94^{\mathrm{de}}$ & $35.92^{\mathrm{cd}}$ & $90.93^{\mathrm{ab}}$ \\
\hline A & $79.91 \pm 0.5^{\mathrm{g}}$ & $13.86 \pm 0.7^{\text {ef }}$ & $0.21 \pm 0.0^{\mathrm{a}}$ & $1.76 \pm 0.01^{\mathrm{ef}}$ & $6.23 \pm 1.1^{\mathrm{b}}$ & $1.30 \pm 0.3^{\mathrm{b}}$ & $0.26 \pm 0.0^{\text {cd }}$ & $0.96 \pm 0.0^{\mathrm{de}}$ & $86.14 \pm 0.6^{\mathrm{bc}}$ & $-6.63^{\text {ef }}$ & $33.52^{e f}$ & $83.01^{\mathrm{c}}$ \\
\hline B-PR & $83.52 \pm 0.5^{\text {cde }}$ & $13.46 \pm 0.3^{\mathrm{fg}}$ & $0.04 \pm 0.0^{\text {cd }}$ & $1.88 \pm 0.07^{\mathrm{de}}$ & $3.01 \pm 0.3^{\text {cde }}$ & $0.36 \pm 0.2^{\mathrm{d}}$ & $0.04 \pm 0.0^{\mathrm{e}}$ & $0.97 \pm 0.0^{\text {bcd }}$ & $86.54 \pm 0.3^{\mathrm{ab}}$ & $-7.82^{b}$ & $38.78^{\mathrm{b}}$ & $88.87^{\mathrm{ab}}$ \\
\hline B-SP & $84.56 \pm 0.5^{\mathrm{abc}}$ & $13.87 \pm 0.2^{\text {ef }}$ & $0.04 \pm 0.0^{\mathrm{cd}}$ & $1.37 \pm 0.13^{\mathrm{gh}}$ & $1.57 \pm 1.5^{\mathrm{ef}}$ & $1.22 \pm 0.2^{\mathrm{bc}}$ & $0.04 \pm 0.0^{\mathrm{e}}$ & $0.96 \pm 0.0^{\mathrm{d}}$ & $86.13 \pm 0.2^{\mathrm{bc}}$ & $-6.38^{\text {fg }}$ & $32.15^{\text {fg }}$ & $88.92^{\mathrm{ab}}$ \\
\hline B-MG & $85.66 \pm 0.5^{\mathrm{a}}$ & $12.31 \pm 0.5^{\mathrm{g}}$ & $0.09 \pm 0.0^{\mathrm{b}}$ & $2.93 \pm 0.05^{\mathrm{a}}$ & $2.03 \pm 0.0^{\text {def }}$ & $1.67 \pm 0.0^{\mathrm{ab}}$ & $0.22 \pm 0.0^{\text {cd }}$ & $0.97 \pm 0.0^{\mathrm{bcd}}$ & $87.69 \pm 0.4^{\mathrm{a}}$ & $-8.53^{\mathrm{a}}$ & $43.17^{\mathrm{a}}$ & $89.94^{\mathrm{ab}}$ \\
\hline $\mathrm{F}$ & $81.72 \pm 0.2^{\mathrm{f}}$ & $14.62 \pm 0.2^{\text {def }}$ & $0.05 \pm 0.0^{\text {cd }}$ & $2.11 \pm 0.01^{\mathrm{cd}}$ & $3.66 \pm 0.2^{\mathrm{c}}$ & $1.36 \pm 0.3^{\mathrm{b}}$ & $0.26 \pm 0.0^{\mathrm{c}}$ & $0.96 \pm 0.0^{\text {de }}$ & $85.38 \pm 0.1^{\mathrm{bcd}}$ & $-5.17^{\mathrm{i}}$ & $27.27^{i}$ & $91.80^{\mathrm{a}}$ \\
\hline $\mathrm{F}$ & $82.79 \pm 0.2^{\text {def }}$ & $15.42 \pm 0.3^{\mathrm{cd}}$ & $0.04 \pm 0.0^{\mathrm{cd}}$ & $1.94 \pm 0.04^{\mathrm{de}}$ & $1.79 \pm 0.2^{\mathrm{def}}$ & $1.32 \pm 0.3^{b}$ & $0.18 \pm 0.0^{\mathrm{d}}$ & $0.96 \pm 0.0^{\mathrm{cd}}$ & $84.58 \pm 0.3^{\mathrm{de}}$ & $-5.80^{\mathrm{h}}$ & $29.45^{\mathrm{h}}$ & $91.69^{\mathrm{a}}$ \\
\hline $\mathrm{F}$ & $79.45 \pm 0.8^{g}$ & $14.60 \pm 0.1^{\text {def }}$ & $0.03 \pm 0.0^{\mathrm{cd}}$ & $2.10 \pm 0.09^{\mathrm{cd}}$ & $5.95 \pm 0.8^{\mathrm{b}}$ & $2.01 \pm 0.0^{\mathrm{a}}$ & $0.21 \pm 0.0^{\text {cd }}$ & $0.95 \pm 0.0^{\mathrm{de}}$ & $85.40 \pm 0.1^{\mathrm{bcd}}$ & $-6.02^{\text {gh }}$ & $31.64^{\mathrm{g}}$ & $89.84^{\mathrm{ab}}$ \\
\hline $\mathrm{F}$ & $71.64 \pm 0.9^{\mathrm{h}}$ & $18.32 \pm 0.4^{\mathrm{a}}$ & $0.05 \pm 0.0^{\mathrm{cd}}$ & $1.82 \pm 0.08^{\mathrm{de}}$ & $10.04 \pm 0.3^{\mathrm{a}}$ & $1.46 \pm 0.2^{\mathrm{ab}}$ & $0.02 \pm 0.0^{\mathrm{e}}$ & $0.94 \pm 0.0^{\mathrm{e}}$ & $81.68 \pm 0.4^{\mathrm{g}}$ & $-6.31^{\mathrm{fg}}$ & $34.70^{\text {de }}$ & $89.33^{\mathrm{ab}}$ \\
\hline C.V. (\%) & 0.51 & 2.70 & 18.68 & 5.44 & 15.44 & 18.86 & 10.58 & 0.77 & 0.46 & 4.87 & 3.85 & 4.32 \\
\hline
\end{tabular}

Results presented by the average of the triplicates followed \pm standard deviation. Color presents the average values for a six-fold. The values in the same column, with equal letters, were not significant at the 5\% level. Tags: DDE (Defatted Dry Extract); Aw (Water activity); TDE (Total Dry Extract); C.V. (Coefficient of Variation). 
The literature is scarce for butters, moreover, most countries do not have a specific regulation to evaluate the quality of this product. According to the studies found, it was possible to perceive some similarity with the present study, showing that there is no standardization in the manufacture of butters, and that do not meet the parameters required by the technical regulations. A study carried out with butters produced in the state of Khartoum, Sudan, and butters imported from Egypt and New Zealand, found that the physical-chemical parameters were not in accordance with local legislation, and the samples had mainly humidity levels above recommended (16\%) (Ahmed, 2011).

In 120 samples of butters marketed in Iran and compared to the required quality protocols in the country, the results showed that $91.7 \%$ of the samples were outside the Iranian national quality standard (Ghasemloy et al., 2017).

Eight brands of butters marketed in the state of Minas Gerais, Brazil, moisture contents varied from $13.69 \%$ to $30.47 \%$, fat from $67.58 \%$ to $84.32 \%$, chlorides from $0.01 \%$ to $0.24 \%$, ashes from $0.13 \%$ to $5.12 \%$, DDE from $1.92 \%$ to $3.52 \%$ and acidity from 0.84 to $4.54 \mathrm{mmol} / 100 \mathrm{~g}$ (Fernandes et al., 2012), showing irregularities in all parameters when compared to the Brazilian RTIQ.

Analyzing ten different brands of butters commercialized in the city of Viçosa, in the state of Minas Gerais, Brazil, the moisture content varied from $11.51 \%$ to $23.97 \%$, fat from $68.36 \%$ to $85.85 \%$, DDE from $0.56 \%$ and $6.25 \%$ and the acidity ranged from 0.94 to $4.11 \mathrm{~mL} / 100 \mathrm{~g}$, the chloride contents found for common butter ranged from $0.45 \%$ to $1.98 \%$ and for extra butter of $1.21 \%$ to $2.88 \%$ (Silva et al., 2009), where butters were found in disagreement with the Brazilian RTIQ. Since, for butters to be marketed in Brazil, they must comply with the requirements of the RTIQ for butter (Brasil, 1996), and the studies showed irregularities in the minimum quality parameters of that product, which would prevent the sale of butters that do not meet these requirements for minimum and maximum levels of moisture, fat, DDE and acidity.

All 29 samples from this study, salted and unsalted, presented adequate values for acidity, as required by Brazilian legislation, RTIQ. The water activity of the samples evaluated presented an average of 0.96 for the samples salted and unsalted, being considered a normal value for butters (Table 1 and 2).

The values found for color, corroborate with the results found in the study by Méndez-Cid et al. (2017), which evaluated the color of butter during storage for nine months. The results showed high brightness value $\left(\mathrm{L}^{*}\right)$ and indication for the direction of the green color, according to the coordinate $\mathrm{a}^{*}$, and direction to yellow according to the coordinate $\mathrm{b}^{*}$.

In all analyses, for salted and unsalted butters, there was a statistically significant difference in all the parameters, which determines the great challenge between the samples, being associated with the raw material and the production. There are factors that influence the characteristics and composition of milk and milk fatty acids, such as cow's milk, genetic factors, seasonal factors and regional factors (Staniewski et al., 2021; Canbay et al., 2017).

Among the fatty acids detected, C16: 0 (palmitic acid), C18: 1n9 (cis-9,12) (oleic acid), C14: 0 (myristic acid) and C18: 0 (stearic acid) in the composition of the salted and unsalted butters were evaluated in this study. However, there was a significant difference in the fatty acid content between the samples (Tables 3, 4, 5 and 6). 
Table 3. Profile of fatty acids (g/100 g) in samples of national and imported salted butters.

\begin{tabular}{|c|c|c|c|c|c|c|c|c|c|c|c|}
\hline Samples & C4:0 & C6:0 & C8:0 & C10:0 & C12:0 & C14:0 & C14:1 & C15:0 & C16:0 & C16:1 & C17:0 \\
\hline B-RS-S & $2.09 \pm 0.03^{\text {bode }}$ & $3.07 \pm 0.11^{\mathrm{a}}$ & $2.02 \pm 0.07^{\mathrm{a}}$ & $5.37 \pm 0.09^{\mathrm{a}}$ & $6.01 \pm 0.06^{\mathrm{a}}$ & $21.15 \pm 0.06^{\mathrm{a}}$ & $1.47 \pm 0.05^{\mathrm{a}}$ & $2.03 \pm 0.06^{\mathrm{a}}$ & $60.78 \pm 1.22^{\mathrm{a}}$ & $2.56 \pm 0.06^{\mathrm{b}}$ & $0.93 \pm 0.14^{\mathrm{ab}}$ \\
\hline B-PR-S & $2.67 \pm 0.12^{\mathrm{a}}$ & $2.44 \pm 0.29^{\mathrm{ab}}$ & $1.27 \pm 0.39^{\mathrm{bc}}$ & $2.87 \pm 0.81^{\mathrm{b}}$ & $3.41 \pm 0.85^{\mathrm{bc}}$ & $12.86 \pm 3.03^{\mathrm{b}}$ & $1.03 \pm 0.27^{\mathrm{ab}}$ & $1.27 \pm 0.35^{\mathrm{b}}$ & $36.41 \pm 4.90^{\mathrm{b}}$ & $1.76 \pm 0.43^{\mathrm{b}}$ & $0.72 \pm 0.21^{\mathrm{b}}$ \\
\hline B-PR-S & $2.14 \pm 0.03^{\text {bode }}$ & $2.01 \pm 0.13^{\mathrm{bc}}$ & $1.25 \pm 0.42^{\mathrm{bc}}$ & $2.37 \pm 0.68^{\mathrm{bc}}$ & $2.73 \pm 0.79^{\mathrm{bcd}}$ & $10.13 \pm 1.03^{\mathrm{bc}}$ & $0.78 \pm 0.21^{\mathrm{bc}}$ & $0.96 \pm 0.28^{\mathrm{bc}}$ & $29.42 \pm 2.46^{\mathrm{bcd}}$ & $1.96 \pm 0.18^{\mathrm{a}}$ & $0.53 \pm 0.16^{b}$ \\
\hline B-RS-S & $1.70 \pm 0.15^{\text {efg }}$ & $1.61 \pm 0.02^{\text {cde }}$ & $1.06 \pm 0.04^{\mathrm{bc}}$ & $2.87 \pm 0.09^{\mathrm{bc}}$ & $2.58 \pm 0.12^{\mathrm{bcd}}$ & $9.90 \pm 0.49^{\mathrm{bc}}$ & $0.78 \pm 0.03^{\mathrm{bc}}$ & $0.99 \pm 0.04^{\mathrm{bc}}$ & $28.79 \pm 1.46^{\mathrm{bcd}}$ & $1.34 \pm 0.04^{\mathrm{b}}$ & $0.55 \pm 0.02^{\mathrm{b}}$ \\
\hline B-MG-S & $1.17 \pm 0.20^{\mathrm{h}}$ & $1.23 \pm 0.19^{\mathrm{e}}$ & $0.83 \pm 0.12^{\mathrm{bc}}$ & $1.97 \pm 0.23^{\mathrm{c}}$ & $2.12 \pm 0.36^{\mathrm{cd}}$ & $8.51 \pm 1.53^{\mathrm{d}}$ & $0.69 \pm 0.08^{\mathrm{bc}}$ & $0.86 \pm 0.13^{\mathrm{bc}}$ & $13.53 \pm 1.16^{\mathrm{cd}}$ & $1.19 \pm 0.12^{\mathrm{b}}$ & $0.22 \pm 0.01^{\mathrm{a}}$ \\
\hline B-SP-S & $1.78 \pm 0.16^{\mathrm{def}}$ & $1.73 \pm 0.30^{\text {cde }}$ & $1.08 \pm 0.18^{\mathrm{bc}}$ & $2.37 \pm 0.46^{\mathrm{bc}}$ & $2.79 \pm 0.51^{\mathrm{bcd}}$ & $10.84 \pm 1.96^{\mathrm{bc}}$ & $0.97 \pm 0.17^{\mathrm{bc}}$ & $1.14 \pm 0.19^{b}$ & $12.80 \pm 1.27^{\mathrm{dd}}$ & $1.51 \pm 0.26^{\mathrm{b}}$ & $0.65 \pm 0.11^{\mathrm{b}}$ \\
\hline F-S & $1.79 \pm 0.47^{\text {def }}$ & $2.02 \pm 0.65^{\text {bod }}$ & $1.33 \pm 0.45^{\mathrm{b}}$ & $3.06 \pm 1.13^{b}$ & $3.58 \pm 13.31^{\text {bc }}$ & $12.50 \pm 2.61^{\mathrm{b}}$ & $1.13 \pm 0.41^{\mathrm{ab}}$ & $1.06 \pm 0.38^{\mathrm{bc}}$ & $37.29 \pm 1.36^{\mathrm{b}}$ & $1.76 \pm 0.64^{b}$ & $0.53 \pm 0.18^{b}$ \\
\hline B-SP-S & $1.83 \pm 0.09^{\text {cde }}$ & $1.84 \pm 0.16^{\text {bode }}$ & $1.18 \pm 0.12^{\mathrm{bc}}$ & $2.59 \pm 0.37^{\mathrm{bc}}$ & $2.98 \pm 0.35^{\mathrm{bcd}}$ & $10.63 \pm 1.23^{\mathrm{bc}}$ & $0.91 \pm 0.10^{\mathrm{bc}}$ & $1.04 \pm 0.14^{\mathrm{bc}}$ & $31.34 \pm 3.81^{\mathrm{bc}}$ & $1.42 \pm 0.17^{\mathrm{b}}$ & $0.55 \pm 0.08^{b}$ \\
\hline B-RS-S & $1.81 \pm 0.02^{\text {cdef }}$ & $1.73 \pm 0.03^{\text {bode }}$ & $1.12 \pm 0.08^{\mathrm{bc}}$ & $2.26 \pm 0.03^{\mathrm{bc}}$ & $2.67 \pm 0.03^{\mathrm{bcd}}$ & $10.18 \pm 0.11^{\mathrm{bc}}$ & $0.72 \pm 0.05^{\mathrm{bc}}$ & $0.94 \pm 0.02^{\mathrm{bc}}$ & $28.79 \pm 1.02^{\mathrm{bcd}}$ & $1.30 \pm 0.03^{b}$ & $0.50 \pm 0.03^{b}$ \\
\hline F-S & $2.35 \pm 0.09^{\mathrm{ab}}$ & $2.20 \pm 0.09^{\mathrm{bod}}$ & $1.35 \pm 0.06^{\mathrm{bc}}$ & $3.06 \pm 0.05^{\mathrm{b}}$ & $3.66 \pm 0.05^{b}$ & $11.45 \pm 0.08^{b}$ & $1.08 \pm 0.08^{\mathrm{ab}}$ & $1.06 \pm 0.06^{\mathrm{bc}}$ & $32.06 \pm 0.06^{\mathrm{bc}}$ & $1.53 \pm 0.04^{\mathrm{b}}$ & $0.55 \pm 0.04^{\mathrm{b}}$ \\
\hline F-S & $2.19 \pm 0.01^{\mathrm{bcd}}$ & $2.24 \pm 0.05^{\mathrm{bc}}$ & $1.37 \pm 0.05^{\mathrm{b}}$ & $3.05 \pm 0.04^{\mathrm{b}}$ & $3.64 \pm 0.05^{\mathrm{bc}}$ & $11.62 \pm 0.03^{b}$ & $1.14 \pm 0.04^{\mathrm{ab}}$ & $1.14 \pm 0.04^{b}$ & $31.53 \pm 0.06^{\mathrm{bc}}$ & $1.64 \pm 0.05^{\mathrm{b}}$ & $0.56 \pm 0.05^{\mathrm{b}}$ \\
\hline B-RS-S & $2.27 \pm 0.04^{\mathrm{abc}}$ & $1.98 \pm 0.04^{\mathrm{bod}}$ & $1.14 \pm 0.04^{\mathrm{bc}}$ & $2.48 \pm 0.02^{\mathrm{bc}}$ & $2.93 \pm 0.04^{\mathrm{bcd}}$ & $9.89 \pm 0.01^{\mathrm{bc}}$ & $0.86 \pm 0.05^{\mathrm{bc}}$ & $1.03 \pm 0.05^{\mathrm{bc}}$ & $27.14 \pm 0.06^{\mathrm{bcd}}$ & $1.37 \pm 0.04^{\mathrm{b}}$ & $0.56 \pm 0.04^{b}$ \\
\hline A-S & $1.28 \pm 0.02^{\mathrm{gh}}$ & $1.17 \pm 0.05^{\mathrm{e}}$ & $0.66 \pm 0.05^{\mathrm{c}}$ & $1.43 \pm 0.03^{\mathrm{c}}$ & $1.65 \pm 0.03^{\mathrm{d}}$ & $5.51 \pm 0.08^{\mathrm{cd}}$ & $0.53 \pm 0.01^{\mathrm{c}}$ & $0.56 \pm 0.02^{\mathrm{c}}$ & $14.76 \pm 0.05^{\mathrm{cd}}$ & $0.84 \pm 0.05^{b}$ & $0.35 \pm 0.01^{b}$ \\
\hline F-S & $1.35 \pm 0.05^{\text {foh }}$ & $1.56 \pm 0.08^{\mathrm{de}}$ & $0.97 \pm 0.04^{\mathrm{bc}}$ & $1.96 \pm 0.05^{\mathrm{bc}}$ & $2.36 \pm 0.06^{\mathrm{bcd}}$ & $7.59 \pm 0.09^{\mathrm{bc}}$ & $0.75 \pm 0.04^{\mathrm{bc}}$ & $0.74 \pm 0.04^{\mathrm{bc}}$ & $9.26 \pm 0.06^{\mathrm{d}}$ & $1.14 \pm 0.03^{b}$ & $0.40 \pm 0.01^{\mathrm{b}}$ \\
\hline
\end{tabular}

Results presented by the average of the triplicates followed \pm Standard Deviation. The values in the same column, with equal letters, were not significant at the $5 \%$ level.

Table 4. Profile of fatty acids (g/100 g) in samples of national and imported salted butters.

\begin{tabular}{|c|c|c|c|c|c|c|c|c|c|c|}
\hline Samples & C18:0 & $\begin{array}{c}\mathrm{C} 18: 1 n 9 \\
(\text { cis-9,12) }\end{array}$ & $\begin{array}{c}\text { C18:1n9 } \\
(\text { trans-9, 12) }\end{array}$ & $\begin{array}{r}\text { C18:2n6 } \\
(\text { cis-9, 12) }\end{array}$ & $\begin{array}{c}\mathrm{C} 18: 2 n 6 \\
(\text { trans-9,12) }\end{array}$ & C18:3n6 & C18:3n3 & C20:0 & C20:1n9 & CLA \\
\hline B-RS-S & $7.92 \pm 0.27^{\mathrm{a}}$ & $38.82 \pm 0.93^{\mathrm{a}}$ & $3.62 \pm 0.05^{\mathrm{abc}}$ & $4.60 \pm 0.90^{\mathrm{a}}$ & $0.10 \pm 0.01^{\mathrm{a}}$ & $0.17 \pm 0.03^{\mathrm{a}}$ & $0.70 \pm 0.01^{\mathrm{a}}$ & $0.35 \pm 0.04^{\mathrm{a}}$ & $0.96 \pm 0.06^{\mathrm{a}}$ & $0.67 \pm 0.04^{\mathrm{ab}}$ \\
\hline B-PR-S & $14.53 \pm 0.38^{\mathrm{ab}}$ & $26.08 \pm 5.59^{\mathrm{b}}$ & $3.62 \pm 0.98^{\mathrm{abc}}$ & $2.11 \pm 0.56^{\mathrm{b}}$ & $0.10 \pm 0.02^{\mathrm{a}}$ & $0.14 \pm 0.04^{\mathrm{ab}}$ & $0.39 \pm 0.09^{b}$ & $0.29 \pm 0.08^{\mathrm{a}}$ & $0.49 \pm 0.24^{\mathrm{bc}}$ & $0.53 \pm 0.01^{\mathrm{e}}$ \\
\hline B-PR-S & $11.94 \pm 0.38^{\mathrm{bc}}$ & $22.04 \pm 3.86^{\mathrm{bc}}$ & $2.96 \pm 0.83^{\text {bed }}$ & $1.95 \pm 0.54^{\mathrm{b}}$ & $0.47 \pm 0.17^{\text {bod }}$ & $0.10 \pm 0.03^{\text {abcde }}$ & $0.30 \pm 0.07^{\mathrm{bc}}$ & $0.18 \pm 0.04^{\mathrm{b}}$ & $0.53 \pm 0.12^{\mathrm{bc}}$ & $0.58 \pm 0.03^{\text {cde }}$ \\
\hline B-RS-S & $11.84 \pm 0.59^{\mathrm{bc}}$ & $20.73 \pm 1.09^{\mathrm{bc}}$ & $2.91 \pm 0.15^{\mathrm{bcd}}$ & $0.97 \pm 0.06^{\mathrm{b}}$ & $0.27 \pm 0.00^{\mathrm{cd}}$ & $0.09 \pm 0.02^{\text {bcde }}$ & $0.28 \pm 0.08^{\mathrm{bc}}$ & $0.16 \pm 0.01^{\mathrm{bc}}$ & $0.37 \pm 0.06^{\mathrm{bc}}$ & $0.52 \pm 0.01^{\mathrm{e}}$ \\
\hline B-MG-S & $7.83 \pm 0.91^{\mathrm{bc}}$ & $14.29 \pm 0.26^{\mathrm{bc}}$ & $2.75 \pm 0.26^{\mathrm{cd}}$ & $1.65 \pm 0.24^{\mathrm{b}}$ & $0.30 \pm 0.02^{\mathrm{cd}}$ & $0.11 \pm 0.00^{\mathrm{abcd}}$ & $0.24 \pm 0.01^{\mathrm{bc}}$ & $0.14 \pm 0.01^{\mathrm{bc}}$ & $0.36 \pm 0.00^{\mathrm{bc}}$ & $0.70 \pm 0.02^{\mathrm{ab}}$ \\
\hline B-SP-S & $11.89 \pm 0.21^{\mathrm{bc}}$ & $21.79 \pm 0.39^{\mathrm{bc}}$ & $2.85 \pm 0.55^{\mathrm{cd}}$ & $1.49 \pm 0.25^{\mathrm{b}}$ & $0.42 \pm 0.09^{\text {bod }}$ & $0.11 \pm 0.03^{\mathrm{abc}}$ & $0.30 \pm 0.05^{\mathrm{bc}}$ & $0.16 \pm 0.03^{\mathrm{bc}}$ & $0.39 \pm 0.04^{\mathrm{bc}}$ & $0.62 \pm 0.02^{\text {bed }}$ \\
\hline F-S & $9.63 \pm 0.35^{\mathrm{bc}}$ & $19.71 \pm 0.73^{\mathrm{bc}}$ & $1.74 \pm 0.71^{\mathrm{d}}$ & $1.90 \pm 0.77^{\mathrm{b}}$ & $0.68 \pm 0.16^{\mathrm{b}}$ & $0.07 \pm 0.02^{\text {cdef }}$ & $0.31 \pm 0.01^{\mathrm{bc}}$ & $0.13 \pm 0.04^{\mathrm{bc}}$ & $0.39 \pm 0.17^{\mathrm{bc}}$ & $0.69 \pm 0.01^{\mathrm{ab}}$ \\
\hline B-SP-S & $10.95 \pm 1.25^{\mathrm{bc}}$ & $20.51 \pm 0.23^{\mathrm{bc}}$ & $2.04 \pm 0.33^{\mathrm{d}}$ & $2.02 \pm 0.23^{\mathrm{b}}$ & $0.54 \pm 0.00^{\mathrm{bc}}$ & $0.08 \pm 0.01^{\text {bodef }}$ & $0.24 \pm 0.02^{\mathrm{bc}}$ & $0.14 \pm 0.02^{\mathrm{bc}}$ & $0.66 \pm 0.21^{\mathrm{ab}}$ & $0.73 \pm 0.01^{\mathrm{a}}$ \\
\hline B-RS-S & $6.79 \pm 0.56^{\mathrm{c}}$ & $11.77 \pm 0.96^{\mathrm{c}}$ & $2.54 \pm 0.01^{\mathrm{cd}}$ & $0.97 \pm 0.07^{\mathrm{b}}$ & $0.37 \pm 0.00^{\text {cd }}$ & $0.10 \pm 0.02^{\text {abcde }}$ & $0.26 \pm 0.03^{\mathrm{bc}}$ & $0.17 \pm 0.01^{\mathrm{b}}$ & $0.32 \pm 0.05^{\mathrm{c}}$ & $0.66 \pm 0.04^{\mathrm{abc}}$ \\
\hline F-S & $8.56 \pm 0.58^{\mathrm{bc}}$ & $17.47 \pm 0.06^{\mathrm{bc}}$ & $4.24 \pm 0.46^{\mathrm{ab}}$ & $1.64 \pm 0.05^{\mathrm{b}}$ & $0.29 \pm 0.01^{\mathrm{cd}}$ & $0.04 \pm 0.00^{\text {ef }}$ & $0.25 \pm 0.04^{\mathrm{bc}}$ & $0.13 \pm 0.06^{\mathrm{bc}}$ & $0.62 \pm 0.09^{\mathrm{bc}}$ & $0.66 \pm 0.05^{\mathrm{abc}}$ \\
\hline F-S & $9.14 \pm 0.49^{\mathrm{bc}}$ & $18.45 \pm 0.05^{\mathrm{bc}}$ & $4.46 \pm 0.13^{\mathrm{a}}$ & $1.63 \pm 0.05^{\mathrm{b}}$ & $0.34 \pm 0.05^{\mathrm{cd}}$ & $0.02 \pm 0.00^{\mathrm{f}}$ & $0.31 \pm 0.01^{\mathrm{bc}}$ & $0.13 \pm 0.01^{\mathrm{bc}}$ & $0.45 \pm 0.04^{\mathrm{bc}}$ & $0.54 \pm 0.04^{\text {de }}$ \\
\hline B-RS-S & $8.78 \pm 0.51^{\mathrm{bc}}$ & $17.67 \pm 0.04^{\mathrm{bc}}$ & $4.47 \pm 0.07^{\mathrm{a}}$ & $1.76 \pm 0.05^{\mathrm{b}}$ & $0.35 \pm 0.02^{\mathrm{cd}}$ & $0.02 \pm 0.00^{\mathrm{f}}$ & $0.31 \pm 0.05^{\mathrm{bc}}$ & $0.12 \pm 0.01^{\mathrm{bc}}$ & $0.58 \pm 0.09^{\mathrm{bc}}$ & $0.65 \pm 0.03^{\mathrm{abc}}$ \\
\hline A-S & $6.08 \pm 0.02^{\mathrm{c}}$ & $12.50 \pm 0.05^{\mathrm{c}}$ & $3.07 \pm 0.05^{\mathrm{abcd}}$ & $1.56 \pm 0.05^{\mathrm{b}}$ & $0.30 \pm 0.01^{\mathrm{cd}}$ & $0.02 \pm 0.01^{\mathrm{f}}$ & $0.27 \pm 0.01^{\mathrm{bc}}$ & $0.76 \pm 0.05^{\mathrm{c}}$ & $0.42 \pm 0.02^{\mathrm{bc}}$ & $0.51 \pm 0.01^{\mathrm{e}}$ \\
\hline F-S & $5.64 \pm 0.07^{\mathrm{c}}$ & $11.96 \pm 0.10^{c}$ & $2.66 \pm 0.46^{\mathrm{cd}}$ & $1.11 \pm 0.07^{\mathrm{b}}$ & $0.20 \pm 0.01^{\mathrm{d}}$ & $0.04 \pm 0.00^{\text {def }}$ & $0.20 \pm 0.01^{\mathrm{c}}$ & $0.84 \pm 0.04^{\mathrm{bc}}$ & $0.36 \pm 0.05^{\mathrm{bc}}$ & $0.31 \pm 0.02^{\mathrm{f}}$ \\
\hline
\end{tabular}

Results presented by the average of the triplicates followed \pm Standard Deviation. The values in the same column, with equal letters, were not significant at the $5 \%$ level. 
Table 5. Profile of fatty acids (g/100 g) in samples of national and imported unsalted butters.

\begin{tabular}{|c|c|c|c|c|c|c|c|c|c|c|c|}
\hline Samples & C4:0 & C6:0 & C8:0 & C10:0 & C12:0 & C14:0 & C14:1 & C15:0 & C16:0 & C16:1 & C17:0 \\
\hline B-RS & $2.07 \pm 0.09^{\mathrm{bc}}$ & $2.58 \pm 0.28^{\mathrm{bc}}$ & $1.68 \pm 0.11^{\mathrm{cd}}$ & $4.23 \pm 0.22^{\mathrm{cd}}$ & $4.82 \pm 0.25^{b}$ & $16.67 \pm 0.87^{\mathrm{b}}$ & $1.21 \pm 0.06^{\mathrm{cd}}$ & $1.56 \pm 0.01^{\mathrm{bod}}$ & $43.92 \pm 2.18^{\mathrm{cd}}$ & $1.95 \pm 0.10^{\text {cde }}$ & $0.72 \pm 0.05^{\text {bcde }}$ \\
\hline B-SP & $1.82 \pm 0.03^{\mathrm{cd}}$ & $1.77 \pm 0.08^{\mathrm{ef}}$ & $1.01 \pm 0.12^{\mathrm{fg}}$ & $2.79 \pm 0.01^{\text {gh }}$ & $3.05 \pm 0.11^{\mathrm{d}}$ & $11.25 \pm 0.38^{\mathrm{d}}$ & $0.80 \pm 0.09^{\mathrm{fg}}$ & $1.03 \pm 0.01^{\text {gh }}$ & $30.88 \pm 1.04^{\mathrm{fg}}$ & $1.46 \pm 0.03^{\text {figh }}$ & $0.53 \pm 0.01^{\text {fgh }}$ \\
\hline B-PR & $1.66 \pm 0.15^{\mathrm{de}}$ & $2.42 \pm 0.31^{\mathrm{bc}}$ & $1.75 \pm 0.16^{\mathrm{bc}}$ & $4.53 \pm 0.44^{\mathrm{bc}}$ & $5.36 \pm 0.33^{\mathrm{a}}$ & $10.27 \pm 1.65^{\mathrm{e}}$ & $1.30 \pm 0.16^{\mathrm{bcd}}$ & $1.50 \pm 0.24^{\text {cde }}$ & $50.42 \pm 2.97^{\mathrm{bc}}$ & $2.27 \pm 0.21^{\mathrm{abcd}}$ & $0.76 \pm 0.12^{\text {bcd }}$ \\
\hline I & $2.20 \pm 0.01^{\mathrm{b}}$ & $2.98 \pm 0.12^{\mathrm{ab}}$ & $2.10 \pm 0.01^{\mathrm{ab}}$ & $5.51 \pm 0.16^{\mathrm{a}}$ & $6.07 \pm 0.03^{\mathrm{a}}$ & $21.42 \pm 0.46^{\mathrm{a}}$ & $1.57 \pm 0.01^{\mathrm{ab}}$ & $1.98 \pm 0.03^{\mathrm{a}}$ & $62.14 \pm 2.40^{\mathrm{a}}$ & $2.73 \pm 0.46^{\mathrm{a}}$ & $0.97 \pm 0.01^{\mathrm{a}}$ \\
\hline B-SP & $2.06 \pm 0.28^{\mathrm{bc}}$ & $2.70 \pm 0.34^{\mathrm{bc}}$ & $1.96 \pm 0.21^{\mathrm{ab}}$ & $5.04 \pm 0.43^{\mathrm{ab}}$ & $5.99 \pm 0.40^{\mathrm{a}}$ & $18.95 \pm 2.26^{\mathrm{ab}}$ & $1.56 \pm 0.13^{\mathrm{ab}}$ & $1.71 \pm 0.16^{\mathrm{abc}}$ & $57.82 \pm 3.43^{\mathrm{ab}}$ & $2.30 \pm 0.34^{\mathrm{abc}}$ & $0.86 \pm 0.08^{\mathrm{abc}}$ \\
\hline B-RS & $2.23 \pm 0.14^{\mathrm{b}}$ & $2.65 \pm 0.16^{\mathrm{bc}}$ & $1.72 \pm 0.11^{\mathrm{cd}}$ & $4.35 \pm 0.24^{\mathrm{bcd}}$ & $4.90 \pm 0.20^{\mathrm{b}}$ & $17.01 \pm 0.50^{\mathrm{bc}}$ & $1.25 \pm 0.07^{\mathrm{cd}}$ & $1.55 \pm 0.10^{\mathrm{bod}}$ & $45.30 \pm 1.41^{\mathrm{cd}}$ & $2.05 \pm 0.09^{\mathrm{cd}}$ & $0.81 \pm 0.05^{\mathrm{abcc}}$ \\
\hline B-MG & $2.62 \pm 0.01^{\mathrm{a}}$ & $3.37 \pm 0.07^{\mathrm{a}}$ & $2.12 \pm 0.07^{\mathrm{a}}$ & $5.28 \pm 0.13^{\mathrm{ab}}$ & $6.20 \pm 0.29^{\mathrm{a}}$ & $21.07 \pm 0.98^{\mathrm{a}}$ & $1.65 \pm 0.13^{\mathrm{a}}$ & $1.90 \pm 0.10^{\mathrm{ab}}$ & $60.77 \pm 4.53^{\mathrm{a}}$ & $2.69 \pm 0.25^{\mathrm{ab}}$ & $0.90 \pm 0.02^{\mathrm{ab}}$ \\
\hline A & $2.32 \pm 0.05^{\mathrm{ab}}$ & $2.40 \pm 0.28^{\mathrm{bcd}}$ & $1.47 \pm 0.11^{\mathrm{d}}$ & $3.35 \pm 0.38^{\mathrm{efg}}$ & $3.90 \pm 0.42^{\mathrm{cd}}$ & $14.05 \pm 0.15^{\text {cd }}$ & $1.00 \pm 0.09^{\mathrm{def}}$ & $1.34 \pm 0.13^{\text {defg }}$ & $38.31 \pm 4.07^{\mathrm{def}}$ & $1.86 \pm 0.06^{\text {cdef }}$ & $0.75 \pm 0.08^{\text {bcd }}$ \\
\hline B-PR & $1.82 \pm 0.08^{\mathrm{cd}}$ & $1.85 \pm 0.23^{\mathrm{def}}$ & $0.99 \pm 0.19^{\mathrm{fg}}$ & $2.22 \pm 0.49^{\mathrm{h}}$ & $2.61 \pm 0.60^{\mathrm{ef}}$ & $9.98 \pm 2.32^{\mathrm{ef}}$ & $0.82 \pm 0.18^{\mathrm{fg}}$ & $0.96 \pm 0.20^{\mathrm{hi}}$ & $29.03 \pm 6.14^{\text {gh }}$ & $1.37 \pm 0.29^{\text {fgh }}$ & $0.54 \pm 0.11^{\mathrm{efg}}$ \\
\hline B-SP & $2.25 \pm 0.04^{\mathrm{ab}}$ & $2.32 \pm 0.01^{\mathrm{cde}}$ & $1.47 \pm 0.07^{\mathrm{de}}$ & $3.32 \pm 0.14^{\mathrm{efg}}$ & $3.74 \pm 0.19^{d}$ & $14.61 \pm 0.79^{\mathrm{cd}}$ & $1.09 \pm 0.06^{\text {cdef }}$ & $1.39 \pm 0.05^{\text {cdef }}$ & $41.67 \pm 2.30^{\text {cde }}$ & $1.90 \pm 0.10^{\text {cde }}$ & $0.82 \pm 0.04^{\mathrm{abcc}}$ \\
\hline B-MG & $2.19 \pm 0.26^{\mathrm{bc}}$ & $2.14 \pm 0.09^{\text {cde }}$ & $1.31 \pm 0.04^{\mathrm{ef}}$ & $2.78 \pm 0.09^{\text {gh }}$ & $3.15 \pm 0.13^{\mathrm{de}}$ & $11.98 \pm 0.41^{\mathrm{de}}$ & $0.87 \pm 0.04^{\text {efg }}$ & $1.19 \pm 0.05^{\text {efgh }}$ & $34.27 \pm 0.87^{\text {efg }}$ & $1.51 \pm 0.06^{\mathrm{efg}}$ & $0.70 \pm 0.02^{\text {cdef }}$ \\
\hline $\mathrm{F}$ & $2.04 \pm 0.01^{\mathrm{bc}}$ & $2.44 \pm 0.01^{\mathrm{bc}}$ & $1.57 \pm 0.06^{\mathrm{de}}$ & $3.93 \pm 0.18^{\text {def }}$ & $4.74 \pm 0.20^{\mathrm{bc}}$ & $15.88 \pm 0.54^{\mathrm{bc}}$ & $1.34 \pm 0.04^{\mathrm{abc}}$ & $1.37 \pm 0.05^{\text {cdefg }}$ & $46.49 \pm 1.30^{\text {cd }}$ & $2.21 \pm 0.11^{\mathrm{bcd}}$ & $0.65 \pm 0.011^{\text {defig }}$ \\
\hline $\mathrm{F}$ & $1.29 \pm 0.01^{\mathrm{e}}$ & $1.88 \pm 0.04^{\mathrm{f}}$ & $0.76 \pm 0.13^{\mathrm{g}}$ & $0.76 \pm 0.07^{\mathrm{i}}$ & $2.12 \pm 0.41^{\mathrm{f}}$ & $7.15 \pm 1.22^{\mathrm{f}}$ & $0.65 \pm 0.11^{\mathrm{g}}$ & $0.68 \pm 0.13^{\mathrm{i}}$ & $20.44 \pm 3.66^{\mathrm{hi}}$ & $1.02 \pm 0.17^{\text {gh }}$ & $0.32 \pm 0.06^{\mathrm{i}}$ \\
\hline $\mathrm{F}$ & $2.00 \pm 0.07^{\mathrm{bcd}}$ & $1.81 \pm 0.25^{\mathrm{ef}}$ & $1.25 \pm 0.10^{\mathrm{ef}}$ & $3.11 \pm 0.15^{\text {fgh }}$ & $3.64 \pm 0.16^{\mathrm{d}}$ & $11.97 \pm 0.53^{\mathrm{de}}$ & $1.13 \pm 0.05^{\text {cde }}$ & $1.06 \pm 0.05^{\text {fgh }}$ & $32.85 \pm 1.65^{\mathrm{efg}}$ & $1.78 \pm 0.10^{\mathrm{def}}$ & $0.49 \pm 0.02^{\text {ghi }}$ \\
\hline F & $2.35 \pm 0.04^{\mathrm{ab}}$ & $2.53 \pm 0.06^{\mathrm{bc}}$ & $1.44 \pm 0.05^{\mathrm{de}}$ & $3.15 \pm 0.05^{\text {fgh }}$ & $3.68 \pm 0.10^{\mathrm{d}}$ & $11.66 \pm 0.06^{\mathrm{de}}$ & $1.20 \pm 0.10^{\mathrm{cd}}$ & $1.16 \pm 0.05^{\text {efgh }}$ & $15.64 \pm 0.05^{\mathrm{i}}$ & $0.97 \pm 0.05^{\mathrm{h}}$ & $0.34 \pm 0.04^{\mathrm{hi}}$ \\
\hline
\end{tabular}

Results presented by the average of the triplicates followed \pm Standard Deviation. The values in the same column, with equal letters, were not significant at the $5 \%$ level.

Table 6. Profile of fatty acids $(\mathrm{g} / 100 \mathrm{~g})$ in samples of national and imported unsalted butters.

\begin{tabular}{|c|c|c|c|c|c|c|c|c|c|c|}
\hline Samples & C18:0 & $\begin{array}{c}\mathrm{C} 18: 1 n 9 \\
(\text { cis }-9,12) \\
\end{array}$ & $\begin{array}{c}\text { C18:1n9 } \\
(\text { trans-9,12) }\end{array}$ & $\begin{array}{r}\text { C18:2n6 } \\
(\text { cis-9,12) } \\
\end{array}$ & $\begin{array}{c}\mathrm{C} 18: 2 n 6 \\
(\text { trans-9,12) }\end{array}$ & C18:3n6 & C18:3n3 & C20:0 & $\mathrm{C} 20: 1 n 9$ & CLA \\
\hline B-RS & $18.22 \pm 0.96^{\mathrm{abc}}$ & $31.10 \pm 1.66^{\mathrm{bc}}$ & $3.57 \pm 0.39^{\mathrm{abc}}$ & $3.04 \pm 0.17^{\mathrm{def}}$ & $0.65 \pm 0.31^{\text {bcde }}$ & $0.13 \pm 0.02^{\mathrm{b}}$ & $0.63 \pm 0.05^{\mathrm{b}}$ & $0.24 \pm 0.01^{\mathrm{bc}}$ & $0.51 \pm 0.12^{\text {bod }}$ & $0.73 \pm 0.00^{\mathrm{a}}$ \\
\hline B-SP & $15.77 \pm 0.40^{\mathrm{c}}$ & $21.17 \pm 0.04^{\mathrm{e}}$ & $2.76 \pm 0.00^{\text {cde }}$ & $2.33 \pm 0.06^{\mathrm{fgh}}$ & $0.52 \pm 0.00^{\text {cde }}$ & $0.09 \pm 0.00^{\mathrm{b}}$ & $0.35 \pm 0.01^{\mathrm{b}}$ & $0.17 \pm 0.00^{\mathrm{de}}$ & $0.40 \pm 0.10^{\text {cde }}$ & $0.66 \pm 0.03^{\mathrm{abcd}}$ \\
\hline B-PR & $20.01 \pm 1.34^{\mathrm{ab}}$ & $34.74 \pm 3.72^{\mathrm{ab}}$ & $4.38 \pm 0.35^{\mathrm{a}}$ & $3.82 \pm 0.27^{\mathrm{bcd}}$ & $0.94 \pm 0.14^{\mathrm{ab}}$ & $0.16 \pm 0.01^{\mathrm{b}}$ & $0.55 \pm 0.04^{\mathrm{b}}$ & $0.25 \pm 0.03^{\mathrm{bc}}$ & $0.65 \pm 0.14^{\text {bod }}$ & $0.64 \pm 0.04^{\text {bode }}$ \\
\hline I & $20.26 \pm 0.66^{\mathrm{ab}}$ & $38.71 \pm 0.50^{b}$ & $3.64 \pm 0.15^{\mathrm{abc}}$ & $4.75 \pm 0.21^{\mathrm{a}}$ & $0.99 \pm 0.02^{\mathrm{a}}$ & $0.16 \pm 0.00^{\mathrm{b}}$ & $0.72 \pm 0.00^{\mathrm{ab}}$ & $0.33 \pm 0.01^{\mathrm{a}}$ & $0.93 \pm 0.12^{\mathrm{b}}$ & $0.68 \pm 0.03^{\mathrm{abc}}$ \\
\hline B-SP & $18.03 \pm 2.04^{\mathrm{abc}}$ & $35.82 \pm 2.39^{\mathrm{ab}}$ & $3.41 \pm 0.43^{\text {abcd }}$ & $4.14 \pm 0.31^{\mathrm{ab}}$ & $0.72 \pm 0.08^{\mathrm{abcd}}$ & $0.13 \pm 0.01^{\mathrm{b}}$ & $1.87 \pm 0.14^{\mathrm{a}}$ & $0.22 \pm 0.03^{\mathrm{cd}}$ & $0.59 \pm 0.04^{\mathrm{bcd}}$ & $0.59 \pm 0.00^{\mathrm{de}}$ \\
\hline B-RS & $18.43 \pm 0.64^{\mathrm{abc}}$ & $32.80 \pm 0.80^{\mathrm{b}}$ & $4.17 \pm 0.22^{\mathrm{a}}$ & $3.23 \pm 0.12^{\mathrm{cde}}$ & $0.82 \pm 0.09^{\mathrm{abc}}$ & $0.13 \pm 0.00^{\mathrm{b}}$ & $0.64 \pm 0.05^{\mathrm{b}}$ & $0.29 \pm 0.05^{\mathrm{ab}}$ & $0.85 \pm 0.23^{b}$ & $0.60 \pm 0.01^{\text {cde }}$ \\
\hline B-MG & $20.96 \pm 0.05^{\mathrm{a}}$ & $39.00 \pm 1.62^{\mathrm{a}}$ & $4.11 \pm 0.14^{\mathrm{a}}$ & $4.83 \pm 0.38^{\mathrm{a}}$ & $0.96 \pm 0.02^{\mathrm{ab}}$ & $0.15 \pm 0.00^{\mathrm{b}}$ & $0.53 \pm 0.01^{\mathrm{b}}$ & $0.25 \pm 0.00^{\mathrm{bc}}$ & $0.83 \pm 0.05^{\mathrm{bc}}$ & $0.59 \pm 0.00^{\text {cde }}$ \\
\hline A & $17.45 \pm 1.90^{\mathrm{bc}}$ & $30.38 \pm 3.63^{\mathrm{bcd}}$ & $3.71 \pm 0.75^{\mathrm{abc}}$ & $3.93 \pm 0.62^{\mathrm{bc}}$ & $0.54 \pm 0.02^{\text {cde }}$ & $0.13 \pm 0.03^{\mathrm{b}}$ & $0.74 \pm 0.09^{\mathrm{ab}}$ & $0.24 \pm 0.02^{\mathrm{bc}}$ & $2.49 \pm 0.40^{\mathrm{a}}$ & $0.59 \pm 0.00^{\text {cde }}$ \\
\hline B-PR & $10.87 \pm 2.21^{\mathrm{d}}$ & $19.59 \pm 4.62^{\mathrm{e}}$ & $2.87 \pm 0.62^{\text {bcde }}$ & $1.46 \pm 0.36^{\mathrm{ij}}$ & $0.46 \pm 0.05^{\mathrm{de}}$ & $0.10 \pm 0.02^{\mathrm{b}}$ & $0.30 \pm 0.05^{\mathrm{b}}$ & $0.16 \pm 0.03^{\mathrm{de}}$ & $0.39 \pm 0.07^{\mathrm{de}}$ & $0.61 \pm 0.01^{\text {bede }}$ \\
\hline B-SP & $18.30 \pm 1.03^{\mathrm{abc}}$ & $31.71 \pm 1.79^{b}$ & $3.73 \pm 0.21^{\mathrm{abc}}$ & $2.52 \pm 0.09^{\text {efg }}$ & $0.63 \pm 0.00^{\text {bcde }}$ & $0.13 \pm 0.01^{\mathrm{b}}$ & $0.42 \pm 0.02^{\mathrm{b}}$ & $0.26 \pm 0.01^{\mathrm{bc}}$ & $0.59 \pm 0.04^{\text {bod }}$ & $0.69 \pm 0.01^{\mathrm{ab}}$ \\
\hline B-MG & $15.77 \pm 0.55^{\mathrm{c}}$ & $24.79 \pm 0.74^{\text {cde }}$ & $3.85 \pm 0.18^{\mathrm{ab}}$ & $1.98 \pm 0.08^{\mathrm{ghi}}$ & $0.56 \pm 0.06^{\mathrm{cde}}$ & $1.02 \pm 0.88^{\mathrm{a}}$ & $0.37 \pm 0.02^{b}$ & $0.20 \pm 0.01^{\mathrm{cd}}$ & $0.06 \pm 0.00^{\mathrm{e}}$ & $0.69 \pm 0.06^{\mathrm{ab}}$ \\
\hline
\end{tabular}


Table 6. Continued...

\begin{tabular}{ccccccccccc}
\hline$F$ & $11.65 \pm 0.28^{\mathrm{d}}$ & $23.94 \pm 0.68^{\text {de }}$ & $2.20 \pm 0.02^{\mathrm{e}}$ & $2.36 \pm 0.07^{\text {fgh }}$ & $0.71 \pm 0.07^{\text {abcde }}$ & $0.10 \pm 0.01^{\mathrm{b}}$ & $0.42 \pm 0.01^{\mathrm{b}}$ & $0.16 \pm 0.01^{\text {de }}$ & $0.52 \pm 0.03^{\text {bed }}$ & $0.67 \pm 0.04^{\text {abcd }}$ \\
\hline $\mathrm{F}$ & $5.35 \pm 0.96^{\mathrm{ef}}$ & $11.64 \pm 1.75^{\mathrm{f}}$ & $1.13 \pm 0.16^{\mathrm{f}}$ & $11.82 \pm 1.94^{\mathrm{j}}$ & $0.38 \pm 0.09^{\mathrm{e}}$ & $0.04 \pm 0.01^{\mathrm{b}}$ & $0.20 \pm 0.02^{\mathrm{b}}$ & $0.09 \pm 0.00^{\mathrm{f}}$ & $0.87 \pm 0.13^{\mathrm{b}}$ & $0.57 \pm 0.04^{\mathrm{e}}$ \\
\hline $\mathrm{F}$ & $8.68 \pm 0.52^{\mathrm{de}}$ & $20.68 \pm 1.12^{\mathrm{e}}$ & $1.91 \pm 0.11^{\mathrm{ef}}$ & $2.07 \pm 0.05^{\mathrm{ghi}}$ & $0.43 \pm 0.18^{\mathrm{de}}$ & $0.07 \pm 0.00^{\mathrm{b}}$ & $0.37 \pm 0.00^{\mathrm{b}}$ & $0.13 \pm 0.00^{\mathrm{ef}}$ & $0.39 \pm 0.02^{\mathrm{de}}$ & $0.56 \pm 0.02^{\mathrm{e}}$ \\
\hline $\mathrm{F}$ & $4.68 \pm 0.10^{\mathrm{f}}$ & $18.56 \pm 0.05^{\mathrm{e}}$ & $2.41 \pm 0.43^{\mathrm{de}}$ & $1.59 \pm 0.09^{\mathrm{hij}}$ & $0.48 \pm 0.03^{\mathrm{de}}$ & $0.15 \pm 0.04^{\mathrm{b}}$ & $0.31 \pm 0.02^{\mathrm{b}}$ & $0.17 \pm 0.01^{\text {de }}$ & $0.55 \pm 0.06^{\text {bed }}$ & $0.55 \pm 0.05^{\mathrm{e}}$ \\
\hline
\end{tabular}

Results presented by the average of the triplicates followed \pm Standard Deviation. The values in the same column, with equal letters, were not significant at the $5 \%$ level.

The CLA content found in the 29 samples evaluated could corroborate with that detected by other authors, mentioned next, and for the samples of butter with salt, the contents varied from $0.33 \%$ to $0.73 \%$, and for the samples of butter without salt, contents between $0.55 \%$ and $0.73 \%$ were detected (Table 4 and 6 ).

Samet-Bali et al. (2009) developed and evaluated traditional Tunisian salted butter. The average fat content was $65.7 \%$, being lower than the minimun content found in the present study, chlorides $0.29 \%$, acidity $2.25 \mathrm{mmol} / 100 \mathrm{~g}$ and protein $1.10 \%$. The mean water activity was 0.79 , which value is lower than found, which can provide a longer shelf life to the product. The main fatty acids found in the butter samples were C14: 0, C16: 0, C18: 0 and C18: 1n9 (cis-9,12). The values found corroborated with the results presented by this study, as well as in the work of Ledoux et al. (2005), who analyzed 54 French butters for a year, and found that the average annual fat content of butters was $83.6 \%$, mean levels of CLA were $0.45 \mathrm{~g} \mathrm{CLA} / 100 \mathrm{~g}$ in winter butter (butter made from milk produced in January or February), $0.58 \mathrm{~g}$ CLA/100 $\mathrm{g}$ in spring butter (butter made from milk produced in March or April) and $0.80 \mathrm{~g}$ CLA/100 g in summer butter (butter made from milk produced in May, June or July).

These results contribute to explain the statistical difference found in the present study, i.e., the strong regional interference of different races and animal husbandry, and the season of the year in which the milk is obtained and later the butter.

Other studies could confirm these statistical differences, as especially in this one where 16 samples of butters were analyzed, and it was found that the acidity ranged from 0.52 to $0.66 \mathrm{mmol} / 100 \mathrm{~g}$, the humidity from $18.33 \%$ to $19.48 \%$, and the fat contents from $83.5 \%$ to $85.9 \%$; the fatty acids found in the highest concentration in the butter were C16: 0 with $36.4 \%, \mathrm{C} 18$ : $1 \mathrm{n} 9$ (cis-9.12) with $13.6 \%, \mathrm{C} 14: 0$ with $12.5 \%$, C18: 0 with 7,1\% and C4: 0 with 3.5\% (Barros et al., 2013). In a study that analyzed seven brands of butter, the acidity found ranged from 0.76 to $2.03 \mathrm{mmol} / 100 \mathrm{~g}$, the minimum and maximum levels found in the different brands for moisture were $11.31 \%$ and $19.97 \%$, respectively. Chlorides were $0.14 \%$ and $2.21 \%$; the range found for the main fatty acids were as following: $\mathrm{C} 14$ : 0 (9.7\% to $12.1 \%), \mathrm{C} 16$ : 0 (22.0\% to $26.1 \%)$, C18: $0(9.6 \%$ to $11.7 \%)$ and C18: $\ln 9(21.9 \%$ to $24.2 \%)$ (Augusta \& Santana, 1998). The fatty acid profile remains stable in the studies found, varying the content found for each fatty acid.

A study that analyzed eleven samples of commercial butters at six different moments, totaling 66 samples, six of them from Brazil, three from France and two from Argentina, showed that the total lipids varied from $81.8 \%$ to $86.8 \%$, the contents of saturated, monounsaturated and polyunsaturated fatty acids varied from $43.86 \%$ to $52.74 \%$, from $21.65 \%$ to $23.34 \%$ and from $2.11 \%$ to $2.89 \%$, respectively, the CLA levels ranged from $0.56 \%$ to $0.86 \%$ and for saturated fatty acids, the most abundant in the butters evaluated was C16: 0 , C18: 0 and C14: 0 (Gonçalves \& Baggio, 2012). The difference in the CLA content found in the samples presented in the literature and in this study was related to seasonal and regional influences.

Méndez-Cid et al. (2017) developed salted and unsalted butters in France and evaluated the composition and quantification of fatty acids and their alteration during the shelf life of the products. In this study, the total dry extract of the unsalted fresh butter was $84.2 \%$ and with salt of $86.5 \%$, the fat found was $92.0 \%$ for the fresh butter without salt and $90.7 \%$ for the fresh butter with salt. In the butter, immediately after 
manufacture, the fatty acid found in the highest concentration was C16: 0 (33\%), followed by C18: 1n-9 (cis9,12) (24.8\%), C14: 0 (11.5\%), C 18: 0 (9.8\%), C 12: 0 (3.5\%), C 10: 0 (2.8\%), C 4: 0 (2.4\%) and CLA $(0.72 \%)$.

These fatty acids found in butters are related to nutritional benefits, some studies could prove that some components of milk fat may be responsible for the hypocholesterolemic, anticancer and antidiabetic effects (Lopes et al., 2011; Rodríguez-Alcalá et al., 2017). In addition, the intake of a secondary amount of dairy products is known to be inversely associated with metabolic syndrome (Drehmer et al., 2016). Supported by these studies, milk fat is being reevaluated and there has been an increase in interest and consumption of butter considered as a "natural product".

The present study contributed to the knowledge of the characteristics of Brazilian, French, Italian and Argentine butters marketed in Brazil, showing the great difference in the physical-chemical composition that exists among the 29 brands. The results pointed out to a great disregard of commercial butter with the RTIQ of butter, which showed the minimum parameters of product quality, which could lead to the conclusion that there are several butters being marketed in the country that are in disagreement with the legislation that regulates production and marketing.

The discussion is not only focused on banning products, however, on carrying out an evaluation on the quality of national and imported butter, and may be legally allowed, the marketing of butters having different physical-chemical characteristics and maintaining sanitary quality, since the physicochemical alteration of the product can interfere in the microbiological aspects of the butter, which can cause in a shorter shelf life. We consider that this study contributed to the development of our research on butter (Naissinger da Silva et al., 2021), as well as fostering the discussion on the revision of Brazilian legislation and improving inspection, since these butters that are inadequate in relation to the physical-chemical parameters may be of adequate sanitary quality.

The legislation on dairy products has more than 20 years of existence, and the reformulation of the legislation may also contribute to add value to the product, which through modern legislation and that meets new products, butters with additions of special and functional ingredients may be added to the market, such as encapsulated probiotic microorganisms, essential oils, spices and seasonings and even jellies, contributing to add value to the product. In addition, it is necessary to carry out sensory studies, using projective techniques, which have emerged in the last decades as a useful and versatile tool to study consumer needs, beliefs, feelings and motivations, allowing to reveal unconscious motivations or not openly accepted in their behavior purchase (Gambaro, 2018).

\section{Conclusion}

The comparison with Brazilian legislation is extremely important, because it is necessary to identify whether the national and imported products that are being marketed in the country (quality) are acceptable, seeing that quality standards and food laws are designed to meet these requirements. All the analyzed butters presented irregularity with at least one of the mandatory quality parameters of the Brazilian Technical Regulation on Identity and Quality of Butter, thus being fat, humidity or Defatted Dry Extract.

The fatty acids composition of butters also showed a significant statistically differences between the samples, showing that butters are strongly affected by seasonal, regional, breed and animal influences, among others. However, the predominant fatty acids were the same between national and imported brands, such as palmitic acid (C16: 0), oleic acid (C18: 1n9 cis-9), stearic acid (C18: 0) and myristic acid (C14: 0).

This study could expose the importance of a review of the Brazilian legislation regarding the quality parameters of butter, since we emphasized that many butters are being marketed outside the required parameters, however, they are characterized as good quality products that meet the requirements of the market and the consumer. 


\section{Acknowledgements}

Acknowledgement to Coordenação de Aperfeiçoamento de Pessoal de Nível Superior - Brazil (CAPES), financial code 001, for providing scholarships.

\section{References}

Ahmed, S. S. (2011). Characteristics of butter processed in Khartoum state (282 p.). Khartoum: Department of Dairy Production, University of Khartoum in Fulfillment.

Association of Official Analytical - AOAC. (2005). Official methods of analysis of the Association Analytical Chemists (18th ed.). Gaithersburg: AOAC

Augusta, I. M., \& Santana, D. M. N. (1998). Evaluation of the quality of extra type butters sold in the state of Rio de Janeiro. Food Science and Technology, 18(4), 35-41. http://dx.doi.org/10.1590/S0101-20611998000400003

Barros, P. A. V., Glória, M. B. A., Lopes, F. C. F., Gama, M. A. S., Souza, S. M., Mourthé, M. H. F., \& Leão, M. I. (2013). Nutritional quality and oxidative stability of butters produced from the milk of cows fed sugarcane supplemented with sunflower oil. Arquivos Brasileiro de Medicina Veterinária e Zootecnia, 65(5), 1545-1553. https://doi.org/10.1590/S010209352013000500036.

Bligh, E. G., \& Dyer, W. J. (1959). A rapid method of total lipid extraction and purification. Canadian Journal of Biochemistry and Physiology, 37(8), 911-917. PMid:13671378. http://dx.doi.org/10.1139/o59-099

Brasil. (1996, março 11). Aprova os regulamentos técnicos de identidade e qualidade dos produtos lácteos (Portaria $n^{\circ} 146$, de 07/03/1996). Diário Oficial [da] República Federativa do Brasil (p. 3977), Brasília, seção 1.

Brasil. (2017, março 30). Regulamenta a Lei no 1.283, de 18 de dezembro de 1950, e a Lei $n^{\circ} 7.889$, de 23 de novembro de 1989, que dispõem sobre a inspeção industrial e sanitária de produtos de origem animal (Decreto $n^{\circ} 9.013$, de 29 de março de 2017). Diário Oficial [da] República Federativa do Brasil, Brasília.

Canbay, H., Büyükoğlu, T., Canbaz, A., Uyguralp, İ., \& Tuncer, E. (2017). effect of feeding management and seasonal variation on fatty acid composition and tocopherol content of cows' milk in Region of West Mediterranean, Turkey. Karaelmas Fen ve Mühendislik Dergisi, 7(1), 85-91. Retrieved in 2020, August 16, from https://dergipark.org.tr/en/pub/karaelmasfen/issue/57120/805799

Chamberlain, M. B., Veltri, B. C., Taylor, S. J., Pareas, J. W., Jimenez-Flores, R., Juchem, S. O., Getachew, G., \& DePeters, E. J. (2016). Feeding lactating Holstein cows a lipid source high in palmitic acid changes the fatty acid composition and thermal properties of lipids in milk and butter. The Professional Animal Scientist, 32(5), 672-680. http://dx.doi.org/10.15232/pas.201501483

Christie, W. W. (1982). A simple procedure for rapid transmethylation of glicerolipids and cholesterol esters. Journal of Lipid Research, 23(7), 1072-1075. PMid:6897259. http://dx.doi.org/10.1016/S0022-2275(20)38081-0

Dewhurst, R. J., Shingfield, K. J., Lee, M. R. F., \& Scollan, N. D. (2006). Increasing the concentrations of beneficial polyunsaturated fatty acids in milk produced by dairy cows in high-forage systems. Animal Feed Science and Technology, 131(4), 168-206. http://dx.doi.org/10.1016/j.anifeedsci.2006.04.016

Drehmer, M., Pereira, M. A., Schmidt, M. I., Alvim, S., Lotufo, P. A., Luft, V. C., \& Duncan, B. B. (2016). Total and full-fat, but not low-fat, dairy product intakes are inversely associated with metabolic syndrome in adults. The Journal of Nutrition, 146(1), 8189. PMid:26511614. http://dx.doi.org/10.3945/jn.115.220699

Erkaya, T., Urkek, B., Dogru, U., Çetin, B., \& Sengul, M. (2015). Probiotic butter: Stability, free fatty acid composition and some quality parameters during refrigerated storage. International Journal of Dairy Technology, 49, 102-110. http://dx.doi.org/10.1016/j.idairyj.2015.04.011

Fernandes, R. V. B., Botrel, D. A., Souza, V. R., Rocha, V. V., \& Ramires, C. S. (2012). Evaluation of the physical-chemical parameters of butters of the common type. Revista Acadêmica Ciências Agrárias e Ambientais, 10(2), 171-176. Retrieved in 2020, August 16, from http://www2.pucpr.br/reol/pb/index.php/academica?dd1=7694\&dd99=view\&dd98=pb

Gambaro, A. (2018). Projective techniques to study consumer perception of food. Current Opinion in Food Science, 21, 46-50. http://dx.doi.org/10.1016/j.cofs.2018.05.004

Ghasemloy, I. K. H., Hassanzadazar, H., Forouzan, S. H., Banafshehchin, E. I., Mozafarian, E., Aminzare, M., \& Hashemi, M. (2017). A survey on the quality of traditional butters produced in West Azerbaijan province, Iran. International Food Research Journal, 24(1), 327-332.

Gonçalves, M. F. D., \& Baggio, S. R. (2012). Evaluation of quality of butter from different provenance. Food Science and Technology, 32(3), 629-635. http://dx.doi.org/10.1590/S0101-20612012005000091

Hara, A., \& Radin, N. S. (1978). Lipid extraction of tissues of low toxicity solvent. Analytical Biochemistry, 90(1), 420-426. PMid:727482. http://dx.doi.org/10.1016/0003-2697(78)90046-5

Ledoux, M., Chardigny, J.-M., Darbois, M., Soustre, Y., Sébédio, J.-L., \& Laloux, L. (2005). Fatty acid composition of French butters, with special emphasis on conjugated linoleic acid (CLA) isomers. Journal of Food Composition and Analysis, 18(5), 409-425. http://dx.doi.org/10.1016/j.jfca.2004.01.001

Lopes, F. C. F., Gama, M. A. S., \& Ribeiro, C. G. S. (2011). Production of milk with high CLA content. Brazilian experience. In Pereira, L. G. R., Nobre, M. M., Neves, A. L. A., Campos, M. M., Medonça, L. C., Gomide, C. A. M., dos Santos, G. G., \& 
Siqueira, K. B. (Orgs.). Research, development and innovation for the sustainability of dairy cattle (pp. 251-296). Juiz de Fora: Embrapa Gado de Leite.

Méndez-Cid, F. J., Centeno, J. A., Martínez, S., \& Carballo, J. (2017). Changes in the chemical and physical characteristics of cow's milk butter during storage: Effects of temperature and addition of salt. Journal of Food Composition and Analysis, 63, 121132. http://dx.doi.org/10.1016/j.jfca.2017.07.032

Naissinger da Silva, M., Tagliapietra, B. L., \& Richards, N. S. P. S. (2021). Encapsulation, storage viability, and consumer acceptance of probiotic butter. Lebensmittel-Wissenschaft + Technologie, 139, 110536. http://dx.doi.org/10.1016/j.Iwt.2020.110536

Nilchian, Z., Ehsani, M. R., Piravi-Vanak, Z., \& Bakhoda, H. (2020). Comparative analysis of butter thermal behavior in combination with bovine tallow. Food Science and Technology, 40(Suppl. 2), 597-604. http://dx.doi.org/10.1590/fst.32019

Parmar, P., Lopez-Villalobos, N., Tobin, J. T., Murphy, E., Crowley, S. V., Kelly, A. L., \& Shalloo, L. (2021). Development and evaluation of a processing sector model for butter manufacture using a mass balance technique at two dairy processing sites. International Journal of Dairy Technology, 74(1), 192-201. http://dx.doi.org/10.1111/1471-0307.12737

Rodríguez-Alcalá, L. M., Castro-Gómez, M. P., Pimentel, L. L., \& Fontecha, J. (2017). Milk fat components with potential anticancer activity: A review. Bioscience Reports, 37(6), BSR20170705. PMid:29026007. http://dx.doi.org/10.1042/BSR20170705

Samet-Bali, O., Ayadi, M. A., \& Attia, H. (2009). Traditional Tunisian butter: Physical-chemical and microbial characteristics and storage stability of the oil fraction. Lebensmittel-Wissenschaft + Technologie, 42(4), 899-905. http://dx.doi.org/10.1016/j.Iwt.2008.11.007

Santos-Zago, L. F., Botelho, A. P., \& Oliveira, A. V. (2008). Os efeitos do ácido linoléico conjugado no metabolismo animal: Avanço das pesquisas e perspectivas para o futuro. Revista de Nutrição, 21(2), 195-221. http://dx.doi.org/10.1590/S141552732008000200008

Silva, L. C. A., Leite, M. O., Vinha, M. B., Brandão, S. C. C., Pinto, C. L. O., \& Carvalho, A. F. (2009). Study of the quality of butters by alkaline phosphatase and physicalchemical analyses. Revista do Instituto de Latícinios Cândido Tostes, 64(367-368), $42-47$

Soundous, E. H., Amaury, G., Juliette, D. L., Sybille, D. T., Aurélie, L., Viviane, P., \& Marianne, S. (2019). Overview of the local production process of raw milk butter in Wallonia (Belgium). International Journal of Dairy Technology, 72, 466-471. http://dx.doi.org/10.1111/1471-0307.12608

Staniewski, B., Ogrodowska, D., Staniewska, K., \& Kowalik, J. (2021). The effect of triacylglycerol and fatty acid composition on the rheological properties of butter. International Dairy Journal, 114, 104913. http://dx.doi.org/10.1016/j.idairyj.2020.104913

Verruck, S., Balthazar, C. F., Rocha, R. S., Silva, R., Esmerino, E. A., Pimentel, T. C., Freitas, M. Q., Silva, M. C., Cruz, A. G., \& Prudencio, E. S. (2019). Chapter Three - Dairy foods and positive impact on the consumer's health. Advances in Food and Nutrition Research, 89, 95-164. PMid:31351531. http://dx.doi.org/10.1016/bs.afnr.2019.03.002

Visentainer, J. V. (2012). Analytical aspects of the flame ionization detector response for fatty acid esters in biodiesel and food. Quimica Nova, 35(2), 274-279. http://dx.doi.org/10.1590/S0100-40422012000200008

Yang, J., Zheng, N., Wang, J., \& Yang, Y. (2018). Comparative milk fatty acid analysis of different dairy species. International Journal of Dairy Technology, 71(2), 544-550. http://dx.doi.org/10.1111/1471-0307.12443

Zoidis, E., Poulopoulou, I., Tsoufi, V., Massouras, T., \& Hadjigeorgiou, J. (2018). Effects of terpene administration on goats' milk fatty acid profile and coagulation properties. International Journal of Dairy Technology, 2(71), 992-996.

http://dx.doi.org/10.1111/1471-0307.12529

Funding: Coordenação de Aperfeiçoamento de Pessoal de Nível Superior - Brazil (CAPES), financial code 001. 